TRANSACTIONS OF THE

AMERICAN MATHEMATICAL SOCIETY

Volume 183, September 1973

\title{
MULTILINEAR IDENTITIES OF THE MATRIX RING
}

\author{
BY
}

\section{URI LERON}

ABSTRACT. Let $V$ be a vector space over a field $F$ of zero characteristic, which is acted upon by the symmetric group. Systems of generators for $V$ are constructed, which have special symmetry and skew symmetry properties. This is applied to prove that every multilinear polynomial identity of degree $2 n+1$ which holds in the matrix ring $F_{n}(n>2)$ is a consequence of the standard identity $s_{2 n}$. The notions of rigid and semirigid sequences of matrices are defined and treated.

0 . Introduction. Let $f\left(x_{1}, \cdots, x_{k}\right)$ be a polynomial in noncommutative variables over a field $F$ and let $R$ be an $F$-algebra. The polynomial $f$ is an identity in $R$ provided $f\left(a_{1}, \cdots, a_{k}\right)=0$ for all $a_{1}, \cdots, a_{k}$ in $R . R$ is called a Pl-ring if it has some nonzero polynomial for an identity. For instance, the commutative rings satisfy the identity $x y-y x=0$. More ge nerally, Amitsur and Levitzki proved in [1] that the matrix ring of order $n$ over a commutative ring satisfies the identity $s_{2 n}=0$, where $s_{k}\left(x_{1}, \cdots, x_{k}\right)=\Sigma_{\sigma \in \Sigma_{k}}(-1)^{\sigma} x_{\sigma(1)} \cdots x_{\sigma(k)}$ (here $\Sigma_{k}$ denotes the symmetric group on $\{1, \cdots, k\}) . s_{k}$ is called the standard polynomial, or standard identity of degree $k_{.} s_{k}$ is an example of a multilinear polynomial, i.e. a polynomial having the form $f\left(x_{1}, \cdots, x_{k}\right)=\Sigma_{\sigma \in \Sigma_{k}} a_{\sigma} x_{\sigma(1)} \cdots x_{\sigma(k)}$ with $\alpha_{\sigma} \in F$. For a more detailed discussion of the theory of polynomial identities see [2] and [3].

In this work $F$ is assumed throughout to be a field of zero characteristic, and $F_{n}$ denotes the ring of $n \times n$ matrices over $F$. Our main concern here is with the vector spaces $V_{k}$ of all the multilinear identities of $F_{n}$ in the variables $x_{1}, \cdots, x_{k}$. It has been known for a long time that $V_{k}=\{0\}$ for $k<2 n$ (i.e. $F_{n}$ satisfies no nonzero identities of degree less than $2 n$ ) and that $V_{2 n}$ is spanned by the standard identity $s_{2 n}$. It is also known that $F_{n}$ satisfies an identity of degree $1 / 2(n+1)(n+2)-1$ which does not follow from $s_{2 n}$. (This result of Amitsur has not been published.) Here a first attempt is made at classifying the identities of degrees higher that $2 n$. For the simplest case, namely that of degree $2 n+1$ (it turns out that already this case is not at all simple!) we achieve

Presented to the Society, January 28, 1973; received by the editors November 28, 1972.

AMS (MOS) subject classifications (1970). Primary 16A38, 16A42; Secondary 15 A24.

Key words and phrases. Matrix ring, multilinear polynomial, standard identity, rigid and semirigid sequences. 
complete results: It is proved that the space $V_{2 n+1}$ is spanned by identities that follow from $s_{2 n}$, and its dimension is computed.

The machinery developed in the course of proving the above theorem might be useful also in the more general case. We first study general spaces acted upon by the symmetric group and construct for them systems of generators $w$ ith special symmetry and skew symmetry properties $(\$ 1)$. It therefore suffices to prove the main theorem for such generators of $V_{2 n+1}$, and this is done in $\$ \$ 3$ and 4 . In the course of such a proof one requires a lot of substitutions from $F_{n}$ with predetermined properties; $\$ 2$ provides some criteria of combinatorial character which guarantee the existence of such substitutions. In $\$ 5$ the dimension of $V_{2 n+1}$ is computed (it is $4 n(n+1)$ ) and the paper is concluded with an example: an identity in $V_{2 n+1}$ is constructed, which does not seem trivially to follow from $s_{2 n}$; as a matter of fact, we had to resort to the main theorem in order to see this.

Unfortunately, the degree of complexity of the case we have treated permits us little hope for the treatment of higher degrees. Some essential improvement on our techniques seems to be needed.

1. Representation modules for the symmetric group. A vector space $V$ over $F$ which is a module over the group algebra $F\left(\Sigma_{k}\right)$ is called a representation module for $\Sigma_{k}$. Recall that $\Sigma_{k}$ is the symmetric group on $\{1, \cdots, k\}$ and $F$ is a field of zero characteristic. Our chief example of a representation module is the space $V_{k}$ defined in the introduction. The action of $\Sigma_{k}$ on $V_{k}$ is defined by

$$
\pi f\left(x_{1}, \ldots, x_{k}\right)=f\left(x_{\pi(1)}, \ldots, x_{\pi(k)}\right),
$$

or, more explicitly, if $f\left(x_{1}, \cdots, x_{k}\right)=\Sigma_{\sigma \in \Sigma_{k}} a_{\sigma} x_{\sigma(1)} \cdots x_{\sigma(k)}$ then

$$
\pi f=\sum_{\sigma \in \boldsymbol{\Sigma}_{k}} \alpha_{\sigma} x_{\pi \sigma(1)} \cdots x_{\pi \sigma(k)}=\sum_{\sigma \in \Sigma_{k}} a_{\pi^{-1}{ }^{\prime} x_{\sigma(1)}} \cdots x_{\sigma(k)} .
$$

This action induces on $V_{k}$ the structure of $F\left(\Sigma_{k}\right)$-module, thus turning it into a representation module for $\Sigma_{k}$. Note that if $f\left(x_{1}, \cdots, x_{k}\right)$ is an identity for $F_{n}$ then so is $f\left(x_{\pi(1)}, \cdots, x_{\pi(n)}\right)$, that is, $V_{k}$ is closed under the action of $\Sigma_{k}$.

Definition. Let $V$ be a representation module for $\Sigma_{k}$ and suppose an element $v \in V$ and numbers $1 \leq i<j \leq k$ are given. Let $(i j)$ be the transposition in $\Sigma_{k}$ interchanging the numbers $i$ and $j$ and leaving the rest fixed. $v$ is called (ij)symmetric if $(i j) v=v$ and $(i j)$-skew symmetric if $(i j) v=-v$.

For instance, a polynomial $f\left(x_{1}, \cdots, x_{k}\right) \in V_{k}$ is $(i j)$-symmetric if and only if $f\left(\cdots x_{i} \cdots x_{j} \cdots\right)=f\left(\cdots x_{j} \cdots x_{i} \cdots\right)$ and $(i j)$-skew symmetric if and only if $f\left(\cdots x_{i} \cdots x_{j} \cdots\right)=-f\left(\cdots x_{j} \cdots x_{i} \cdots\right)$. In terms of the coefficients $a_{\sigma}$ of $f$, $f$ is $(i j)$-symmetric if and only if $\alpha_{(i j) \sigma}=\alpha_{\sigma}$ for all $\sigma \in \Sigma_{k}$, and $(i j)$-skew symmetric if and only if $\alpha_{(i j) \sigma}=-\alpha_{\sigma}$ for all $\sigma \in \Sigma_{k}$. 
It is convenient to characterize the symmetry and skew symmetry of elements of $V$ using the operator $\sigma_{i j} \in F\left(\Sigma_{k}\right)$, defined as follows:

$$
\sigma_{i j}=1 / 2(1+(i j))
$$

Note that $\sigma_{i j}$ is an idempotent linear transformation in $V$, and that the set of $(i j)$-symmetric $\left((i j)\right.$-skew symmetric) elements is just $\operatorname{Im} \sigma_{i j}\left(\operatorname{ker} \sigma_{i j}\right)$. In other words, $v$ is $(i j)$-symmetric if and only if $\sigma_{i j} v=v$ and (ij)-skew symmetric if and only if $\sigma_{i j} v=0$.

Lemma 1. Let $V$ be a representation module for $\Sigma_{k}$ and suppose an element $v \in V$ and distinct numbers $p, q, r$ from among $1, \cdots, k$ are given. If $\sigma_{p q} v=0$ then $\sigma_{p r} \sigma_{q r} v=1 / 2 \sigma_{p r} v$.

Proof.

$$
\begin{aligned}
\sigma_{p r} \sigma_{q r}-1 / 2 \sigma_{p r} & =1 / 2 \sigma_{p r}(1+(q r))-1 / 2 \sigma_{p r} \\
& =1 / 2 \sigma_{p r}(q r)=1 / 4(1+(p r))(q r)=1 / 4((q r)+(p r)(q r)) \\
& =1 / 4((q r)+(q r)(p q))=1 / 4(q r)(1+(p q))=1 / 2(q r) \sigma_{p q} .
\end{aligned}
$$

Hence $\left(\sigma_{p r} \sigma_{q r}-1 / 2 \sigma_{p r}\right) v=1 / 2(q r) \sigma_{p q} v=0$.

Theorem 2 (Lifting skew symmetric elements). Let $V$ be a representation module for $\Sigma_{k}, U$ an $F$-subspace of $V$. Suppose distinct numbers $i_{1}, \cdots, i_{r}$ from among $1, \cdots, k$ are given, such that $U$ is $\sigma_{i \mu i \nu}$-invariant for all $1 \leq \mu<\nu$ $\leq r$. If $v \in V$ satisfies $\sigma_{i \mu i \nu} \equiv 0(\bmod U)$ for all $1 \leq \mu<\nu \leq r$ then there exists $v^{\prime} \in V$ satisfying $\nu^{\prime} \equiv \nu(\bmod U)$ and $\sigma_{i \mu i} \nu^{\prime}=0$ for all $1 \leq \mu<\nu \leq r$. Moreover, if $v$ is $(i j)$-symmetric where $\{i, j\} \cap\left\{i_{1}, \cdots, i_{r}\right\}=\varnothing_{2}$ then $v^{\prime}$ will remain $(i j)$. symmetric.

Proof. We use induction on $r$, assuming for convenience that $\left\{i_{1}, \cdots, i_{r}\right\}=$ $\{1, \cdots, r\}$. For $r=1, v^{\prime}=v$ will obviously do. Suppose there is already given $v^{\prime \prime} \in V$ satisfying $v^{\prime \prime} \equiv v(\bmod U), \sigma_{p q} \nu^{\prime \prime}=0$ for $1 \leq p<q \leq r-1$ and $\sigma_{i j} v^{\prime \prime}=v^{\prime \prime}$, and proceed to define

$$
v^{\prime}=\left(1-\frac{2}{r} \sum_{p=1}^{r-1} \sigma_{p r}\right) v^{\prime \prime}=v^{\prime \prime}-\frac{2}{r} \sum_{p=1}^{r-1} \sigma_{p r} v^{\prime \prime} .
$$

Since $v^{\prime \prime} \equiv v(\bmod U)$ and $U$ is $\sigma_{p r}$-invariant we have $\sigma_{p r} v^{\prime \prime} \equiv \sigma_{p r} v \equiv 0(\bmod U)$ for $p=1, \cdots, r-1$; hence, by the definition of $v^{\prime}, v^{\prime} \equiv v^{\prime \prime} \equiv v(\bmod U)$. To compute $\sigma_{q r} v^{\prime}=\left(\sigma_{q r}-(2 / r) \Sigma_{p=1}^{r-1} \sigma_{q r} \sigma_{p r}\right) v^{\prime \prime}$, consider the summation on $p$. For $p=q$ we 
get the term $\sigma_{q r}^{2}$ which is just $\sigma_{q r}$. For $p \neq q$ the induction hypothesis gives $\sigma_{p q} v^{\prime \prime}=0$ so that Lemma 1 is applicable, yielding $\sigma_{q r} \sigma_{p r} v^{\prime \prime}=1 / 2 \sigma_{q r} v^{\prime \prime}$. Summing up, we have

$$
\begin{aligned}
\sigma_{q r} \nu^{\prime} & =\left(\sigma_{q r}-\frac{2}{r}\left(\sigma_{q r}+\frac{1}{2} \sum_{p=1, p \neq q}^{r-1} \sigma_{q r}\right)\right) v^{\prime \prime} \\
& =\left(1-\frac{2}{r}-\frac{r-2}{r}\right) \sigma_{q r} v^{\prime \prime}=(1-1) \sigma_{q r} \nu^{\prime \prime}=0 .
\end{aligned}
$$

If now any numbers $1 \leq p<q \leq r$ are given, write $(p q)=(p r)(q r)(p r)$, hence $(p q) v^{\prime}$ $=(p r)(q r)(p r) v^{\prime}=(-1)^{3} v^{\prime}=-v^{\prime}$ and $\sigma_{p q} v^{\prime}=0$. Finally, the disjointness of $\{i, j\}$ and $\{1, \cdots, r\}$ implies that $\sigma_{i j}$ commutes with $a=1-(2 / r) \sum_{p=1}^{r-1} \sigma_{p r}$ and inducting on $r$ one obtains

$$
\sigma_{i j} v^{\prime}=\sigma_{i j} a v^{\prime \prime}=a \sigma_{i j} v^{\prime \prime}=a v^{\prime \prime}=v^{\prime},
$$

that is, $v^{\prime}$ is $(i j)$-symmetric.

Definition. Let $V$ be a representation module for $\Sigma_{k}$ and suppose an element $v \in V$ and a number $r \geq 0$ are given. $v$ is called $r$-symmetric if for some distinct numbers $i_{1}, j_{1} ; i_{2}, j_{2} ; \cdots ; i_{r}, j_{r}$ from among $1, \cdots, k v$ is $\left(i_{\nu} j_{\nu}\right)$-symmetric, $\nu=1, \cdots, r$. If, in addition $v$ is $(p q)$-skew symmetric for all $1 \leq p<q$ $\leq k$ such that $\{p, q\} \cap\left\{i_{1}, j_{1}, \cdots, i_{r}, j_{r}\right\}=\varnothing$ then $v$ is called $r$-perfect.

Note that every $v \in V$ is 0 -s ymmetric. On the other hand, $v$ is 0 -perfect if and only if it is $(p q)$-skew symmetric for all $1 \leq p<q \leq k$. In particular, a polynomial $f \in V_{k}$ is 0 -perfect if and only if it is a scalar mult iple of the standard polynomial $s_{k}$.

Theorem 3. Let $V$ be a representation module for $\Sigma_{k}$ and let $S_{r}$ and $P_{r}$ be the subspaces of $V$ spanned respectively by its r-symmetric and r-perfect ele. ments. Then

(a) $S_{r}=P_{r}+S_{r+1}$ for all $r \geq 0$.

(b) $V=P_{0}+P_{1}+\cdots+P_{r}+S_{r+1}$ for all $r \geq 0$.

(c) $V=P_{0}+P_{1}+\cdots+P_{t}$ for some $t$, that is, $V$ is generated by its perfect elements.

Proof. (a) Clearly $S_{r} \supseteq P_{r}+S_{r+1}$, so we prove the reverse inclusion, noting that it is enough to do so for $r$-symmetric elements only. Let $v \in V$ be $r$-symmetric and assume for convenience that $v$ is (1 2)-, (34)-, $\cdots,(2 r-12 r)$-symmetric. Then, for every $2 r<p<q \leq k, \sigma_{p q} v$ is $(r+1)$-symmetric, i.e., $\sigma_{p q} v \equiv$ $0\left(\bmod S_{r+1}\right)$. Since it is easy to verify that $S_{r+1}$ is $\sigma_{p q}$-invariant (it is enough to show it is (pq)-invariant), Theorem 2 is applicable yielding a $v^{\prime} \in V$ such that 
$v^{\prime} \equiv v\left(\bmod S_{k+1}\right), \sigma_{p q} v^{\prime}=0$ for all $2 r<p<q \leq k$ and $\sigma_{i j} v^{\prime}=v^{\prime}$ for $(i j)=\left(\begin{array}{ll}1 & 2\end{array}\right)$, (3 4), $\cdots,(2 r-1 r)$. But this just means that $v^{\prime} \in P_{k}$ and $v-v^{\prime} \in S_{k+1}$, that is, $v \in P_{k}+S_{k+1}$.

(b) Apply (a) a few times in succesion to get

$$
V=S_{0}=P_{0}+S_{1}=P_{0}+P_{1}+S_{2}=\cdots=P_{0}+P_{1}+\cdots+P_{k}+S_{k+1} .
$$

(c) There exists $t$ for which $S_{t+1}=\{0\}$ and, by (b), this is the $t$ we are after.

2. Rigid sequences. Let $a_{1}, \cdots, a_{k}$ be matrices in $F_{n}$ and let $u=\left(a_{1}\right.$, $\left.\cdots, a_{k}\right)$. The number $k$ is called the length of the sequence $u$ and is denoted $l(u)$. The value of $u$ is the product $v(u)=a_{1} \cdot a_{2} \cdots a_{k}$. The unit matrices are the matrices $e_{i j}$ in $F_{n}$ having 1 in the $(i, j)$ th position and 0 elsewhere. A sequence $u=\left(a_{1}, \cdots, a_{k}\right)$ is called $n$-simple if every $a_{t}$ is some unit matrix and the set of indices of these unit matrices contains at most $n$ distinct numbers. For example, a sequence of unit matrices from $F_{n}$ is always $n$-simple. A sequence is nonvanisbing if it assumes a nonzero value. Clearly, the value of a nonvanishing simple sequence must be a unit matrix.

Definition. Let $u$ be a nonvanishing $n$-simple sequence, $u$ is called $n$-rigid if none of its nontrivial permutations assumes the same value as $u$.

For instance, the sequences $\left(e_{11}, e_{12}, e_{22}, e_{23}, e_{33}\right)$ and $\left(e_{12}, e_{23}, e_{33}\right.$, $\left.e_{32}, e_{21}\right)$ are 3-rigid sequences of length 5 .

It turns out that rigid sequences can be neatly characterized in terms of the derived sequence which we now define. Let $u$ be a nonvanishing $n$-simple sequence. Then $u$ must have the form

$$
u=\left(e_{i_{1} i_{2}}, e_{i_{2} i_{3}}, e_{i_{3} i_{4}}, \cdots, e_{i_{k} i_{k+1}}\right) .
$$

The number sequence $\left(i_{1}, i_{2}, i_{3}, \cdots, i_{k+1}\right)$ will be denoted $u^{\prime}$ and called the derived sequence of $u$. Conversely, every sequence $u^{\prime}=\left(i_{1}, i_{2}, \cdots, i_{k+1}\right)$ such that the set $\left\{i_{1}, \cdots, i_{k+1}\right\}$ has at most $n$ elements defines an $n$-simple sequence $u$ having $u^{\prime}$ as its derived sequence. Note that $l\left(u^{\prime}\right)=l(u)+1$. The derived sequence $u^{\prime}$ of $u$ is called $n$-rigid if and only if $u$ is $n$-rigid. Finally, suppose there is given a number $r$ and a sequence $u^{\prime}=\left(i_{1}, \ldots, i_{k+1}\right)$ such that $r=i_{\nu}$ for some $1 \leq \nu \leq k+1$. We then say that $i_{\nu}$ is an occurrence of $r$ in $u^{\prime}$, or that $r$ occurs in $u^{\prime}$ in the $\nu$ th position. Such an occurrence is external if $\nu=1$ or $\nu=k+1$, and internal otherwise.

The notion of rigidity is of interest mainly for $n$-simple sequences of length $2 n-1$ (i.e. $l\left(u^{\prime}\right)=2 n$ ). This is because the maximal length for which $n$-rigid sequences exist is $2 n-1$, as follows easily from the proof of the next theorem. 
Theorem 4. Let $u$ be a nonvanishing $n$-simple sequence of length $2 n-1$, $u^{\prime}$ its derived sequence. Then $u$ is rigid if and only if the following two conditions hold:

(i) Every number $r$ occurring in $u^{\prime}$ occurs in it exactly twice.

(ii) Denote the first and second occurrences of a number $r$ in $u^{\prime}$ by $i_{\nu_{1}(r)}$ and $i_{\nu_{2}(r)}$ respectively (i.e. $i_{\nu_{1}(r)}=i_{\nu_{2}(r)}=r$ and $\left.\nu_{1}(r)<\nu_{2}(r)\right)$. Then for no $r$ and $s$ does the relation $\nu_{1}(r)<\nu_{1}(s)<\nu_{2}(r)<\nu_{2}(s)$ bold.

We may think of the two positions of occurrence of $r$ in $u^{\prime}$ as a pair of parentheses, thus obtaining the following interpretation for condition (ii). The sequence of parentheses defined by $u^{\prime}$ is well formed, i.e. two pairs of parentheses may be separated $\left({ }_{r}\right)_{r}\left({ }_{s}\right)_{s}$ or nested $\left({ }_{r}\left({ }_{s}\right)_{s}\right)_{r}$, but not interlaced $\left({ }_{r}\left({ }_{s}\right)_{r}\right)_{s}$.

Proof. (a) Suppose $u$ is rigid. To prove (i), assume on the contrary that some $r$ does not occur in $u^{\prime}$ exactly twice. Since $u^{\prime}$ has length $2 n$ and at most $n$ numbers occurring in it, our assumption implies the existence of an $r$ which occurs in $u^{\prime}$ at least 3 times. These three occurrences of $r$ decompose the original sequence $u$ into four blocks, $u=\left(u_{1}, u_{2}, u_{3}, u_{4}\right)$, such that $l\left(u_{1}\right), l\left(u_{4}\right) \geq 0$, $l\left(u_{2}\right), l\left(u_{3}\right) \geq 1$ and $v\left(u_{2}\right)=v\left(u_{3}\right)=e_{r r}$. Thus the blocks $u_{2}$ and $u_{3}$ may be transposed in $u$ without affecting its value-a contradiction. To prove (ii), assume we do have a relation $\nu_{1}(r)<\nu_{1}(s)<\nu_{2}(r)<\nu_{2}(s)$, i.e. $r$ and $s$ occur in $u^{\prime}$ in the order $\cdots r \cdots s \cdots r \cdots s \cdots$. Then $u$ can be decomposed into 5 blocks $u=$ $\left(u_{1}, u_{2}, u_{3}, u_{4}, u_{5}\right)$ such that $l\left(u_{1}\right), l\left(u_{5}\right) \geq 0, l\left(u_{2}\right), l\left(u_{3}\right), l\left(u_{4}\right) \geq 1$ and $v\left(u_{2}\right)=$ $v\left(u_{4}\right)=e_{r s}$. Transposing $u_{2}$ and $u_{4}$ we obtain a contradiction as before.

(b) Suppose $u$ satisfies the condition (i) and (ii) and proceed by induction on $n$ to prove $u$ rigid. For $n=1$ the assertion is trivial. For $n=2$ it is enough to consider sequences $u^{\prime}$ starting, say, with 1 . There are only two such sequences satisfying (i) and (ii), namely $(1,2,2,1)$ and $(1,1,2,2$, ) and the sequences $u$ defined by them, $\left(e_{12}, e_{22}, e_{21}\right)$ and $\left(e_{11}, e_{12}, e_{22}\right)$ are clearly seen to be rigid. For $n \geq 3$ we may find $r$ whose second occurrence in $u^{\prime}$ immediately succeeds the first one, and both are internal. To see this, choose any number $r$ occurring only internally, which is possible since $n \geq 3$ and by (i) at least 3 distinct numbers must occur in $u^{\prime}$. If it happens that $\nu_{2}(r)=\nu_{1}(r)+1$ then we are done. If not, let $s_{1}, \cdots, s_{t}$ be all the numbers having one of their occurrences (hence both by (ii)) between $\nu_{1}(r)$ and $\nu_{2}(r)$, where the notation is so chosen that $\nu_{1}(r)<\nu_{1}\left(s_{1}\right)<\nu_{1}\left(s_{2}\right)<\cdots<\nu_{1}\left(s_{t}\right)<\nu_{2}(r)$. It is then easily seen that the two occurrences of $s_{t}$ are internal and the one immediately succeeds the other. So choose $r$ as above, and note that, in the original sequence $u, r$ occurs inside a block of the form $e_{l r}, e_{r r}, e_{r m}$ and nowhere else. Let $u_{0}$ be the sequence obtained from $u$ by replacing the block $e_{l r}, e_{r r}, e_{r m}$ by the single term 
$e_{l m}$. Then $u_{0}$ is an $(n-1)$-simple sequence ( $r$ does not occur in it) of length $2(n-1)-1$, satisfying (i) and (ii). By the induction hypothesis $u_{0}$ is rigid, from which it easily follows that so is $u$.

Theorem 4 enables us to determine the possibility of constructing a rigid sequence, when the positions of occurrence of some indices are predetermined. This is the content of the next theorem.

Theorem 5. Let there be given distinct numbers $r_{1}, \cdots, r_{k}, k \leq n$, and for each $r_{i}$ let a pair $\nu_{1}\left(r_{i}\right)<\nu_{2}\left(r_{i}\right)$ out of $\{1, \ldots, 2 n\}$ be given, so that all the $\nu_{j}\left(r_{i}\right)$ are distinct. In order that there should exist an n-rigid sequence $u$ of length $2 n-1$ for which the numbers $\nu_{1}\left(r_{i}\right), \nu_{2}\left(r_{i}\right)$ would be the positions of oc. currence of $r_{i}$ in $u^{\prime}$, it is necessary and sufficient that the following two conditions sball bold:

I. The number $\nu_{2}\left(r_{i}\right)-\nu_{1}\left(r_{i}\right)$ is odd for $i=1, \cdots, k$.

II. For no $i$ and $j$ does the relation $\nu_{1}\left(r_{i}\right)<\nu_{1}\left(r_{j}\right)<\nu_{2}\left(r_{i}\right)<\nu_{2}\left(r_{j}\right)$ hold.

Proof. To prove necessity, suppose such $u$ exists, and invoke the conditions of Theorem 4. It follows that each number occurring in $u^{\prime}$ must occur between the two occurrences of a particular $r_{i}$ either twice or not at all. Thus the number $\nu_{2}\left(r_{i}\right)-\nu_{1}\left(r_{i}\right)-1$ of positions between the two positions occupied by $r_{i}$ is even, proving condition I. Condition II is obvious.

We shall now prove that the conditions also suffice, assuming for convenience that $\left\{r_{1}, \cdots, r_{k}\right\}=\{1, \cdots, k\}$. Write $A=\left\{\nu_{j}(r) \mid r=1, \cdots, k, j=1,2\right\}$. We shall call the numbers $1, \cdots, 2 n$ "positions," and describe them as "occupied" or "vacant" according to whether they belong to $A$ or not. Our objective is then to fill in the vacancies so as to obtain a rigid sequence $u^{\prime}$. If there are no vacanies, then the sequence $u^{\prime}$ defined by the given positions $\nu_{j}(r)$ satisfy (i) and (ii) of Theorem 4 and is therefore rigid. Suppose now that the number of vacancies is $>0$ (i.e. $k<n$ ), and note that the number of vacancies between $\nu_{1}(r)$ and $\nu_{2}(r)$ must always be even. For both the number of occupied positions and the total number of positions between $\nu_{1}(r)$ and $\nu_{2}(r)$ is even (the former by II, the latter by I). We start the construction of $u^{\prime}$ by assigning two vacancies for $k+1$ as follows. $\nu_{1}(k+1)$ is defined as the first vacancy, i.e. the smallest number not in $A$. If $\nu_{1}(k+1)+1$ is also vacant, take $\nu_{2}(k+1)=\nu_{1}(k+1)+1$. Suppose, on the other hand, that $\nu_{1}(k+1)+1$ is occupied. We assert that there exists a sequence of numbers $s_{1}, \cdots, s_{t}, 1 \leq s_{i} \leq k$, such that

(1) $\nu_{1}\left(s_{1}\right)=\nu_{1}(k+1)+1$,

(2) $\nu_{1}\left(s_{i}\right)=\nu_{2}\left(s_{i-1}\right)+1, i=2, \cdots, t$,

(3) $\nu_{2}\left(s_{t}\right)+1$ is vacant.

Indeed, since $\nu_{1}(k+1)+1$ is occupied we must have $\nu_{1}(k+1)+1=\nu_{j}\left(s_{1}\right)$ for 
some $1 \leq s_{1} \leq k$. Here $j$ cannot be 2 , for this would imply only one vacancy between $\nu_{1}\left(s_{1}\right)$ and $\nu_{2}\left(s_{1}\right)$ (namely, $\nu_{1}(k+1)$ ), whereas this number is known to be even. Thus $\nu_{1}(k+1)+1=\nu_{1}\left(s_{1}\right)$. If $\nu_{2}\left(s_{1}\right)+1$ is vacant, our assertion is proved taking $t=1$. If not, we have $\nu_{2}\left(s_{1}\right)+1=\nu_{j}\left(s_{2}\right)$ for some $1 \leq s_{2} \leq k$. Again $j$ must be 1 , for suppose $j=2$ and consider $\nu_{1}\left(s_{2}\right)$. By II, $\nu_{1}\left(s_{2}\right)$ cannot be between $\nu_{1}\left(s_{1}\right)$ and $\nu_{2}\left(s_{1}\right)$, hence it must be smaller than $\nu_{1}(k+1)$. The vacancies between $\nu_{1}\left(s_{2}\right)$ and $\nu_{2}\left(s_{2}\right)$ are therefore made up from those between $\nu_{1}\left(s_{1}\right)$ and $\nu_{2}\left(s_{1}\right)$ (an even number) and $\nu_{1}(k+1)$, which is impossible since this number must be even. Thus $\nu_{2}\left(s_{1}\right)+1=\nu_{1}\left(s_{2}\right)$. Continuing in the same manner, one finally arrives at an $1 \leq s_{t} \leq k$ such that either $\nu_{2}\left(s_{t}\right)=2 n$ or $\nu_{2}\left(s_{t}\right)+1$ is vacant. But just as before the number of vacancies smaller than or equal to $\nu_{2}\left(s_{t}\right)$ is odd, whereas the number of vacancies smaller than or equal to $2 n$ is even (namely, $2 n-2 k$ ). So we conclude that $\nu_{2}\left(s_{t}\right)+1$ is vacant, which proves our assertion. We now define $\nu_{2}(k+1)=\nu_{2}\left(s_{t}\right)+1$.

[At this stage, the partially-filled sequence $u^{\prime}$ has the following form: $\left.\left(\cdots, k+1, s_{1}, \cdots, s_{1}, s_{2}, \cdots, s_{2}, \cdots, s_{t}, \cdots, s_{t}, k+1, \cdots\right).\right]$

Let $\bar{A}=\left\{\nu_{j}(r) \mid r=1, \cdots, k+1, j=1,2\right\}$ be the extended set of occupied positions. We assert that $\bar{A}$ still satisfies conditions I and II of the theorem. The case where $\nu_{1}(k+1)+1$ was vacant (and subsequently was defined to be $\left.\nu_{2}(k+1)\right)$ is trivial, so proceed with the other case. The positions between $\nu_{1}(k+1)$ and $\nu_{2}(k+1)$ are

$$
\nu_{1}\left(s_{1}\right), \ldots, \nu_{2}\left(s_{1}\right), \nu_{1}\left(s_{2}\right), \ldots, \nu_{2}\left(s_{2}\right), \ldots, \nu_{1}\left(s_{t}\right), \ldots, \nu_{2}\left(s_{t}\right),
$$

so there is an even number of them and condition $\mathrm{I}$ is proved for $k+1$. For $1 \leq$ $r \leq k, \mathrm{I}$ is easily seen to be preserved. To establish condition II, assume that for some $1 \leq r \leq k$ we have $\nu_{1}(k+1)<\nu_{1}(r)<\nu_{2}(k+1)$. Then, by the construction of $\nu_{j}(k+1)$, we also have $\nu_{1}\left(s_{i}\right)<\nu_{1}(r)<\nu_{2}\left(s_{i}\right)$ for some $1 \leq i \leq t$, forcing $\nu_{1}\left(s_{i}\right)<\nu_{2}(r)<\nu_{2}\left(s_{i}\right)$ since II holds in $A$. Thus $\nu_{1}(k+1)<\nu_{2}(r)<\nu_{2}(k+1)$. Similarly one proves that if $\nu_{2}(r)$ is between $\nu_{1}(k+1)$ and $\nu_{2}(k+1)$ then so is $\nu_{1}(r)$. Since II holds in $A$, what we proved suffices to establish its validity for $\bar{A}$, thus proving our assertion. The number of vacancies is thus reduced by 2 , and induction on this number concludes the proof of the theorem.

To illustrate the general construction given in the proof of Theorem 5, we shall fill in the vacant positions in the following sequence:

$$
u^{\prime}=(1,2,2, \cdot, 3, \cdot, 4,4, \cdot, 3,5, \cdot, \cdot, 5, \cdot, 1),
$$

so as to obtain a rigid sequence. Here $n=8$ and the occupied positions are $\nu_{1}(1)=1, \nu_{2}(1)=16 ; \quad \nu_{1}(2)=2, \nu_{2}(2)=3 ; \nu_{1}(3)=5, \nu_{2}(3)=10 ; \nu_{1}(4)=7$, $\nu_{2}(4)=8 ; \nu_{1}(5)=11, \nu_{2}(5)=14$. The vacant positions are $4,6,9,12,13,15$. 
The general construction first yields $\nu_{1}(6)=4$ and $\nu_{2}(6)=15$ (the sequence $s_{1}, \cdots, s_{t}$ being in this case $s_{1}=3$ and $\left.s_{2}=5\right)$, then $\nu_{1}(7)=6, \nu_{2}(7)=9$ and finally $\nu_{1}(8)=12, \nu_{2}(8)=13$. The complete sequence is

$$
u^{\prime}=(1,2,2,6,3,7,4,4,7,3,5,8,8,5,6,1) \text {. }
$$

Note that if we were given, say, $\nu_{2}(5)=13$ (instead of 14) there would be no way to complete $u^{\prime}$ rigidly.

One application we shall need later is the following

Corollary 6. Let $1 \leq s_{1}<s_{2}<\cdots<s_{t} \leq 2 n-1$ be given numbers such that $s_{i}-s_{i-1}>1$ for $i=2, \cdots, t$. Then there exists an $n$-rigid sequence of length $2 n-1$, baving in its $s_{i} t h$ position an idempotent for all $1 \leq i \leq t$.

Proof. Choose arbitrary distinct numbers $1 \leq r_{1}, \cdots, r_{t} \leq n$ and define a sequence $u^{\prime}=\left(i_{1}, \cdots, i_{2 n}\right)$ by $i_{s_{1}}=i_{s_{1}+1}=r_{1}, \cdots, i_{s_{t}}=i_{s_{t}+1}=r_{t}$. By Theorem 5 , this partial definition of $u^{\prime}$ can be completed rigidly, and the sequence $u$ defined by $u^{\prime}$ satisfies all the requirements.

Numerous additional applications will occur in $\$ \S 3$ and 4 .

So far, we were concerned with $n$-simple sequences of length $2 n-1$, but our main interest in the sequel will be with longer sequences, where rigidity is impossible. To render the above theorems applicable in the more general case we introduce the following term.

Definition. An $n$-simple sequence $u$ is called $n$-semirigid if by deleting a certain number of idempotents, one can obtain from $u$ an $n$-rigid sequence of length $2 n-1$.

For instance, $\left(e_{12}, e_{22}, e_{22}, e_{21}, e_{11}\right)$ is a 2-semirigid sequence of length 5 : By delet ing the idempotents in its $3 \mathrm{rd}$ and 5 th positions, a rigid sequence is obtained.

Semirigid sequences are useful because for them it is easy to determine the value-preserving permutations (i.e. permutations for which the permuted sequence has the same value as the original one). Indeed, let $u$ be semirigid and let $u_{0}$ be a rigid sequence of length $2 n-1$ obtained from $u$ by deleting some idempotents. Suppose

$$
\sigma=\left(\begin{array}{c}
a_{1}, \ldots, a_{k} \\
a_{i_{1}}, \ldots, a_{i_{k}}
\end{array}\right)
$$

is a value-preserving permutation of $u$. Then by ignoring the above idempotents in both rows of this representation, $\sigma$ induces a value-preserving permutation of 
$u_{0}$, which must be the identity since $u_{0}$ is rigid. From this analysis it is seen that the action of $\sigma$ on $u$ can only amount to an interchange of some of the extra idempotents $e_{i i}$ with adjacent blocks having the value $e_{i i}$ (of course the block may be of length 1 , in which case it is just another $e_{i i}$ ).

As an illustration, consider an $n$-semirigid sequence $u$ of length $2 n$. $u$ is obtained from a rigid sequence $u_{0}$ by adding a single $e_{i i}$. Since $u_{0}$ has just one block of value $e_{i i}$ (corresponding to the two occurrences of $i$ in $u_{0}^{\prime}$ ), there is only one nontrivial value-preserving permutation of $u$, namely, that interchanging $e_{i i}$ with the $e_{i i}$-valued block. Similarly it is easy to see that the number of valuepreserving permutations (the identity included) of an $n$-semirigid sequence $u$ of length $2 n+1$ is 4 or 6 , depending on whether $u$ is obtained from $u_{0}$ by adding two distinct or identical idempotents.

Finally, the following observation seems to be of interest. So far the multilinear identities of the matrix ring have been fully characterized in three cases: degree $\leq 2 n-1$ (only the zero polynomial), degree $2 n$ and degree $2 n+1$ (only identities following from the standard identity $s_{2 n}$ ). In all these cases only semirigid substitutions were used in the proof, which leads us to the following conjecture: A multilinear polynomial which vanishes under every semirigid substitution from $F_{n}$ is an identity of $F_{n}$. As indicated above, this conjecture is true for all polynomials of degree $\leq 2 n+1$. To demonstrate the power of the conjecture we point out that it implies almost immediately (with the aid of Theorem 5) the Amitsur-Levitzki theorem mentioned in the introduction.

3. Main theorem: the perfect case. From now on we shall be concerned exclusively with the space $V_{2 n+1}$, so we call it $A$ for short. Recall that $A$ is the vector space of all multilinear identities of $F_{n}$ in the variables $x_{1}, \cdots, x_{2 n+1}$. By the Amitsur-Levitzki theorem, $s_{2 n}$ is an identity of $F_{n}$, hence $A$ obviously contains the following identities:

$$
\begin{aligned}
& \phi_{i}=x_{i} s_{2 n}\left(x_{1}, \ldots, x_{i-1}, x_{i+1}, \ldots, x_{2 n+1}\right), \quad i=1, \ldots, 2 n+1, \\
& \psi_{i}=s_{2 n}\left(x_{1}, \ldots, x_{i-1}, x_{i+1}, \ldots, x_{2 n+1}\right) x_{i}, \quad i=1, \ldots, 2 n+1, \\
& \chi_{i j}=s_{2 n}\left(x_{1}, \ldots, x_{i-1}, x_{i} x_{j}, x_{i+1}, \ldots, x_{j-1}, x_{j+1}, \ldots, x_{2 n+1}\right), \\
& i, j=1, \ldots, 2 n+1, i \neq j .
\end{aligned}
$$

Let $B$ be the subspace of $A$ spanned by these identities. Our main theorem states that, for $n>2, A=B$, i.e. every identity in $A$ follows from the standard identity $s_{2 n}$. The proof is based on the following corollary of Theorem 3 .

Theorem 7. Let $A$ be as above and let $T$ be the set consisting of all the 1-perfect polynomials of $A$, all the 2-symmetric polynomials of $A$ and the stan- 
dard polynomial $s_{2 n+1}$. Then $A$ is spanned by $T$.

Proof. Note first that by the Amitsur-Levitzki theorem $s_{2 n+1}$ is in $A$, for we have $s_{2 n+1}=\phi_{1}-\phi_{2}+\phi_{3}-\phi_{4}+\cdots+\phi_{2 n+1}$. This expansion is analogous to the expansion of a determinant by first row and is easily proved. (What we have actually shown is that $s_{2 n+1} \in B$.) The proof of the theorem now immediately follows from Theorem $3(\mathrm{~b})$, since $A$ is a representation module for $\Sigma_{2 n+1}$ and $P_{0}$ is spanned by $s_{2 n+1}$.

In order to prove the main theorem it is enough by Theorem 7 to prove $T \subseteq B$. Since we have already seen that $s_{2 n+1} \in B$, it is necessary to establish $B$-membership only in the cases of 1-perfect polynomials and 2-symmetric polynomials. The former will be treated in the present section and the latter, which is more involved, in the next. But first we list the results of applying the operators $(k l)$ and $\sigma_{k l}$ of $\S 1$, to the polynomials $\phi_{1}$ and $\chi_{i j}$ defined above. Let $i, j, k, l$ be distinct numbers; then

(a) $(k l) \phi_{i}=-\phi_{i}$ hence $\sigma_{k l} \phi_{i}=0 ;(i k) \phi_{i}=(-1)^{k-i+1} \phi_{i}$ hence $2 \sigma_{i k} \phi_{i}=$ $\phi_{i}-(-1)^{k-i} \phi_{k}$.

(b) $(k l) \chi_{i j}=-\chi_{i j}$ hence $\sigma_{k l} \chi_{i j}=0$; $(i k) \chi_{i j}=-\chi_{k j}$ hence $2 \sigma_{i k} \chi_{i j}=\chi_{i j}-$ $\chi_{k j} ;(k j) \chi_{i j}=(-1)^{k-j+1} \chi_{i k}$ hence $2 \sigma_{k j} \chi_{i j}=\chi_{i j}-(-1)^{k-j} \chi_{i k} ;(i j) \chi_{i j}=$ $(-1)^{i-j+1} \chi_{j i}$ hence $2 \sigma_{i j} \chi_{i j}=\chi_{i j}-(-1)^{i-j} \chi_{j i}$.

Lemma 8., Let $i, j, k, l$ be dist inct numbers.

(a) The following polynomials in $B$ are (kl)-symmetric, i.e. they are fixed by $\sigma_{k l}: \chi_{k j}-\chi_{l j}, \chi_{i k}-(-1)^{k-l} \chi_{i l}, \chi_{k l}-(-1)^{k-l} \chi_{l k}$.

(b) The following polynomials in $B$ are (kl)-skew symmetric, i.e. they are annibilated by $\sigma_{k l}: \chi_{i j}, \chi_{k j}+\chi_{l j}, \chi_{i k}+(-1)^{k-l} \chi_{i l}, \chi_{k l}+(-1)^{k-l} \chi_{l k}$.

The proof of the list and lemma is straightforward and will be omitted.

We now get down to proving that every 1 -perfect identity $f \in A$ is in $B$. It is enough to consider (12)-perfect identities (by which we mean identities that are (12)-symmetric and $(i j)$-skew symmetric for every $2<i<j$ ) for all other 1 -perfect identities can be gotten from these by substituting the variables in different order. For the sake of brevity we therefore agree that in this section the term "perfect identity" will always mean "(12)-perfect identity."

Lemma 9. B contains 4 linearly independent perfect identities.

Proof. Define

$$
\begin{array}{ll}
p_{1}=\phi_{1}+\phi_{2}, & p_{2}=\psi_{1}+\psi_{2}, \\
p_{3}=\chi_{12}+\chi_{21}, & p_{4}=\sum_{i=3}^{2 n+1}\left(\chi_{i 1}+\chi_{i 2}\right) .
\end{array}
$$


Since the standard identity is $(i j)$-skew symmetric for every $i \neq j$, it is clear from the definition of $\phi_{i}, \psi_{i}, X_{i j}$ that $p_{1}, p_{2}, p_{3}$ are perfect and that $p_{4}$ is (12)-symmetric. We have yet to show that $p_{4}$ is ( $\left.k l\right)$-skew symmetric for all $2<k<l$. Indeed, using Lemma 8 (or direct observation) we get

$$
\begin{aligned}
\sigma_{k l} p_{4} & =\sum_{i=3}^{2 n+1} \sigma_{k l}\left(\chi_{i 1}+\chi_{i 2}\right)=\sigma_{k l}\left(\chi_{k 1}+\chi_{k 2}\right)+\sigma_{k l}\left(\chi_{l 1}+\chi_{l 2}\right) \\
& =\sigma_{k l}\left(\chi_{k 1}+\chi_{l 1}\right)+\sigma_{k l}\left(\chi_{k 2}+\chi_{l 2}\right)=0+0=0 .
\end{aligned}
$$

Suppose now $a_{1} p_{1}+a_{2} p_{2}+a_{3} p_{3}+a_{4} p_{4}=0$. Equating to zero the coefficients of the monomials $x_{1} x_{2} \cdots x_{2 n+1}, x_{1} x_{3} x_{2} \cdots x_{2 n+1}, x_{1} x_{3} x_{4} x_{2} \cdots x_{2 n+1}$ and $x_{1} x_{3} x_{4} \cdots x_{2 n+1} x_{2}$ we get the respective equations $a_{1}+a_{3}=0,-a_{1}+$ $a_{4}=0, a_{1}+a_{4}=0$ and $-a_{1}+a_{2}+a_{4}=0$, which immediately yield $a_{1}=a_{2}$ $=a_{3}=a_{4}=0$.

Lemma 10. Let $f \in A$ be a perfect identity in which the monomials $x_{1} x_{2} \cdots$ $x_{2 n+1}, x_{1} x_{3} x_{2} x_{4} \cdots x_{2 n+1}, x_{1} x_{3} x_{4} x_{2} x_{5} \cdots x_{2 n+1}$ and $x_{1} x_{3} \cdots x_{2 n+1} x_{2}$ occur with zero coefficients. Then $f=0$. For

For the proof let us introduce some notation. Let $f=\Sigma_{\sigma \in \Sigma_{2 n+1}} \alpha_{\sigma^{x}}{ }_{\sigma(1)} \cdots x_{(2 n+1)}{ }^{*}$

$$
\sigma=\left(\begin{array}{llll}
1 & 2 & \cdots & 2 n+1 \\
i_{1} i_{2} & \cdots & i_{2 n+1}
\end{array}\right)
$$

we shall write for short $\alpha_{\sigma}=\alpha_{i_{1} i_{2}} \cdots_{i_{2 n+1}}$. Moreover, in a particular equation involving coefficients of $f$, we shall write explicitly only those indices varying their positions along the equation. Thus the equation $\alpha_{12345}+\alpha_{32145}+\alpha_{12435}$ $+a_{42315}=0$, in which the indices 2 and 5 have the same position in all the terms, will be written as $a_{134}+a_{314}+a_{143}+a_{431}=0$.

Proof. Recall that $f$ is (12)-symmetric and $(i j)$-skew symmetric for all $2<$ $i<j$. Therefore if some monomial occurs in $f$ with zero coefficient, so do all the monomials obtained from it by a permutation of $\{3, \cdots, 2 n+1\}$ or $\{1,2\}$. Thus ;in order to prove that every monomial has zero coefficient in $f$, we have only to consider the various possible positions of $x_{1}$ and $x_{2}$ in the monomial, without regard to the order of the other variables. We distinguish several cases.

(a) Monomials in which $x_{2}$ immediately succeeds $x_{1}$ occur in $f$ with zero coefficient. We are given $\alpha_{123} \cdots_{2 n+1}=0$. To prove $\alpha_{3124} \cdots_{2 n+1}=0$, substitute for the variables in $f$ the following $n$-semirigid sequence 


$$
\begin{aligned}
& \begin{array}{llllll}
x_{1} & x_{2} & x_{3} & x_{4} & x_{3} & \cdots
\end{array} \\
& u:\left(e_{11}\right)\left(e_{11}\right) \quad e_{11} \quad e_{12} \quad e_{22} \quad \cdots \text {, }
\end{aligned}
$$

in which the matrix assigned to each variable is written directly under that variable. The parentheses indicate the idempotents whose deletion will result in a rigid sequence of length $2 n-1$ (see $\$ 2$ ). Here and in the sequel we define the substitution only for the relevant variables, and omit the check that this satisfies the conditions of Theorem 5 and thus can be completed to a semirigid substitution. Write $f(u)=\sum_{i, j=1}^{n} t_{i j} e_{i j}=0$ since $f$ is an identity for $F_{n}$, so that $t_{i j}=0$ for all $i, j$. In particular, if the value of $u$ is $e_{r s}$ then $t_{r s}=0$. Taking into account the six value-preserving permutations of $u$, we obtain $t_{r s}=\left(a_{123}+a_{213}\right)+$ $\left(a_{132}+a_{231}\right)+\left(a_{312}+a_{321}\right)=0$. By the (12)-symmetry of $f$, the two terms in each pair of parentheses are equal, and since the characteristic of $F$ is not 2, this implies $a_{123}+a_{132}+a_{312}=0$. But $a_{123}=a_{132}=0$, therefore $a_{312}=$ 0 which is what we set out to prove. Case (a) will now be completed by induction on the position of the block $x_{1} x_{2}$ in the monomial. Suppose it is already known that $a_{3 \cdots i 12 i+1 \cdots 2 n+1}=0(i \geq 2)$. If $i<2 n$ we make the semirigid subsubstitution (again using The orem 5 )

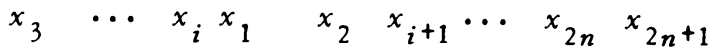

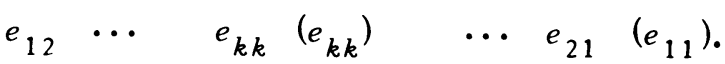

By the (12)-symmetry of $f$ we get as before $a_{3 \cdots i 12 i+1 \cdots 2 n 2 n+1}+$ $a_{2 n+13 \cdots i 12 i+1} \cdots 2 n=0$. The first term vanishes by the induction hypothesis, hence so does the second term. This completes the induction for all $i<2 n$. To prove $\alpha_{3} \cdots 2 n+112=0$, make the semirigid substitution

$$
\begin{array}{ccc}
x_{3} \cdots x_{2 n+1} & x_{1} & x_{2} \\
e_{12} \cdots e_{21} & \left(e_{11}\right) & \left(e_{11}\right)
\end{array}
$$

and conclude that $\alpha_{3 \cdots 2 n+112}+\alpha_{123 \cdots 2 n+1}+\alpha_{13 \cdots 2 n+12}=0$. Since the second and third terms are given to be zero, the first term must also be zero, which completes the proof of case (a).

(b) $x_{1}$ and $x_{2}$ are separated by a single variable, say $x_{3}$. Substitute

$$
\begin{aligned}
& \begin{array}{cccc}
\cdots & x_{1} & x_{3} & x_{2}
\end{array} \cdots \\
& \cdots e_{11}\left(e_{11}\right) \quad\left(e_{11}\right) \ldots
\end{aligned}
$$

(by which we mean, of course, the completion of this assignment to a semirigid sequence; that meaning will be tacitly assumed in all that follows) to get $a_{132}$ 
$+\alpha_{123}+a_{312}=0$. The second and third terms vanish by (a), hence so does the first.

(c) $x_{1}$ and $x_{2}$ are separated by three variables, say $x_{3}, x_{4}, x_{5}$. Substitute

$$
\begin{aligned}
& \begin{array}{lllllll}
\cdots & x_{1} & x_{3} & x_{4} & x_{3} & x_{2} & \cdots
\end{array} \\
& \ldots\left(e_{11}\right) \quad e_{12} \quad e_{22} \quad e_{21} \quad\left(e_{11}\right) \ldots
\end{aligned}
$$

to get $\alpha_{13452}+a_{12345}+\alpha_{34512}=0$. The second and third terms vanish by (a), hence so does the first.

(d) $x_{1}$ and $x_{2}$ are separated by two variables, say $x_{3}, x_{4}$. We shall prove the vanishing of the coefficient by induction on the position of the block $x_{1} x_{3} x_{4} x_{2}$ in the monomial. If the block is in the leftmost position, this is given. In the other case, the monomial has the form $\cdots x_{5} x_{1} x_{3} x_{4} x_{2} \cdots$ and we substitute

$$
\begin{aligned}
& \begin{array}{lllllll}
\cdots & x_{3} & x_{1} & x_{3} & x_{4} & x_{2} & \cdots
\end{array} \\
& \ldots\left(e_{11}\right) \quad e_{11} \quad e_{12} \quad e_{22} \quad\left(e_{22}\right) \ldots \text {, }
\end{aligned}
$$

obtaining $\alpha_{51342}+a_{51324}+a_{15342}+\alpha_{15324}=0$. In this equation the second term vanishes by (b), the third by (c) and the fourth by the induction hypothesis (the block has "moved" one position to the left). Therefore $\alpha_{51342}=0$, as required.

(e) When the number of variables separating $x_{1}$ and $x_{2}$ is greater than 3 , we induct on this number. The monomial in this case has the form $\cdots x_{1} x_{3} x_{4}$ $\cdots x_{5} x_{2} \cdots$ and we substitute

$$
\begin{array}{ccccccc}
\cdots & x_{1} & x_{3} & \cdots & x_{5} & x_{2} & \cdots \\
\cdots & e_{11} & \left(e_{11}\right) & \cdots & e_{22} & \left(e_{22}\right) & \cdots
\end{array}
$$

Such semirigid substitution exists by Corollary 6 , for $x_{3}$ and $x_{5}$ are separated by at least one more variable. The vanishing of $f$ under the substitution gives $a_{1352}+a_{1325}+a_{3152}+a_{3125}=0$. The last three terms vanish by the induction hypothesis, therefore so does the first.

We have proved the vanishing of the coefficient for every possible position of $x_{1}$ and $x_{2}$ in the monomial, thus proving $f$ to be the zero polynomial.

The main theorem of this section now follows easily.

Theorem 11. If $f \in A$ is a perfect identity then $f \in B$.

Proof. Suppose the special monomials from Lemma 10 occur in $f$ with the following coefficients: $\beta_{1} x_{1} x_{2} \cdots x_{2 n+1}, \beta_{2} x_{1} x_{3} x_{2} \cdots x_{2 n+1}, \beta_{3} x_{1} x_{3} x_{4} x_{2} \cdots x_{2 n_{+}}$, 
$\beta_{4} x_{1} x_{3} \cdots x_{2 n+1} x_{2}$. We wish to represent $f$ as a linear combination of the perfect identities $p_{i}$ occurring in Lemma 9. Since $p_{i} \in B$ this would clearly imply $f \in B$. If a representation $f=\alpha_{1} p_{1}+\alpha_{2} p_{2}+\alpha_{3} p_{3}+\alpha_{4} p_{4}$ does exist, we may equate the coefficients of the above special monomials and obtain (compare the proof of Lemma 9): $\alpha_{1}+\alpha_{3}=\beta_{1},-\alpha_{1}+\alpha_{4}=\beta_{2}, \alpha_{1}+\alpha_{4}=\beta_{3}$ and $-\alpha_{1}+$ $a_{2}+a_{4}=\beta_{4}$. Solving for the $\alpha_{i}$ 's we get $\alpha_{1}=1 / 2\left(\beta_{3}-\beta_{2}\right), \alpha_{2}=\beta_{4}-\beta_{2}$, $a_{3}=\beta_{1}-1 / 2\left(\beta_{3}-\beta_{2}\right), \alpha_{4}=1 / 2\left(\beta_{2}+\beta_{3}\right)$. Now define $\alpha_{1}, \alpha_{2}, \alpha_{3}, \alpha_{4}$ by these formulas and let $g=\alpha_{1} p_{1}+\alpha_{2} p_{2}+\alpha_{3} p_{3}+\alpha_{4} p_{4}$. Then both $f$ and $g$ are perfect identities having the same coefficients for the special monomials. Consider $f-g$. This is a perfect identity in which the coefficients of the special monomials vanish; so, by Lemma $10, f-g=0$, i.e. $f=g \in B$.

Incidentally, the last proof (and Lemma 9) shows that $p_{1}, p_{2}, p_{3}, p_{4}$ form a linear basis for the space of (12)-perfect identities in $A$.

4. Main theorem: the 2 -symmetric case. We retain the notation of $\$ 3$. In the present section we prove that every 2-symmetric polynomial in $A$ belongs to $B$, thereby completing the proof of the main theorem. It is enough to prove the assertion for polynomials which are, say, (12)- and (34)-symmetric, so in this section the term "2-symmetric polynomial" will always refer to such a polynomial. Note first that $B$ contains the following two linearly independent 2-symmetric polynomials:

$$
\begin{aligned}
q_{1}= & s_{2 n}\left(x_{1} \cdot x_{3}, x_{2}, x_{4}, \ldots, x_{2 n+1}\right)+s_{2 n}\left(x_{2}, x_{3}, x_{1}, x_{4}, \ldots, x_{2 n+1}\right) \\
& +s_{2 n}\left(x_{1} \cdot x_{4}, x_{2}, x_{3}, \ldots, x_{2 n+1}\right)+s_{2 n}\left(x_{2}, x_{4}, x_{1}, x_{3}, \ldots, x_{2 n+1}\right), \\
q_{2}= & s_{2 n}\left(x_{3} \cdot x_{1}, x_{2}, x_{4}, \ldots, x_{2 n+1}\right)+s_{2 n}\left(x_{3} \cdot x_{2}, x_{1}, x_{4}, \ldots, x_{2 n+1}\right) \\
& +s_{2 n}\left(x_{4} \cdot x_{1}, x_{2}, x_{3}, \ldots, x_{2 n+1}\right)+s_{2 n}\left(x_{4}, x_{2}, x_{1}, x_{3}, \ldots, x_{2 n+1}\right) .
\end{aligned}
$$

We wish to represent every 2-symmetric identity in $A$ as a linear combination of these two by showing, as before, that every 2-symmetric identity in which two special coefficients vanish must be the zero polynomial. Unlike the former case, however, some preparatory work is needed here.

Lemma 12. Let $M_{\sigma}=x_{\sigma(1)} \cdots x_{\sigma(2 n+1)}$ and let $f=\Sigma_{\sigma \in \Sigma_{2 n+1}} a_{\sigma M_{\sigma}} b e(12)$ symmetric identity in $A$. Suppose the monomial $M_{\sigma^{\prime}}$ is obtained from $M_{\sigma}$ by a transposition which involves no immediate neigbbor of $x_{1}$ or $x_{2}$. Then $a_{\sigma}=$ $-a_{\sigma}$

Proof. We may suppose that the transposition is, say, (34) and distinguish four cases according to the relative position of $x_{1}, x_{2}, x_{3}, x_{4}$ in the monomial 
$M_{\sigma}$. Note, however, that at every stage of the proof we may apply the results of former steps also to other pairs satisfying the conditions of the lemma.

(a) Assume that in $M_{\sigma}$ no member of one pair separates the two members of the other pair, say $M_{\sigma}=\cdots x_{1} \cdots x_{2} \cdots x_{3} \cdots x_{4} \cdots$. (If the pair $x_{3}, x_{4}$ occurs to the left of $x_{1}, x_{2}$ or if $x_{4}$ is to the left of $x_{3}$, the treatment is similar.) Suppose first that $x_{4}$ immediately succeeds $x_{3}$ and induct on the distance between $x_{1}$ and $x_{2}$. When the distance is 0 , i.e. $x_{2}$ immediately succeeds $x_{1}, M_{\sigma}$ has the form $M_{\sigma}=\cdots x_{1} x_{2} \cdots x_{3} x_{4} \cdots$, where, by assumption, $x_{2}$ and $x_{3}$ are not immediate neighbors. By Corollary 6 there exists a semirigid substitution of the form

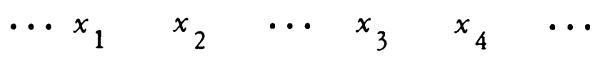

$$
\begin{aligned}
& \cdots e_{11}\left(e_{11}\right) \quad \cdots e_{22} \quad\left(e_{22}\right) \quad \ldots \text {, }
\end{aligned}
$$

which yields (since it annihilates $f$ ) the equation $\alpha_{1234}+\alpha_{2134}+\alpha_{1243}+\alpha_{2143}$ $=0$. Using the (12)-symmetry of $f$ and cancelling out 2 , we get $a_{1234}+a_{1243}$ $=0$, which is the desired conclusion. When the distance between $x_{1}$ and $x_{2}$ is positive, the monomial has the form $M_{\sigma}=\cdots x_{1} x_{5} \cdots x_{2} \cdots x_{3} x_{4} \cdots$, and we make the substitution

$$
\begin{array}{ccccc}
\cdots x_{1} & x_{5} & \cdots x_{2} \cdots & x_{3} & x_{4} \\
\cdots e_{11} & \left(e_{11}\right) & \cdots & e_{22} & \left(e_{22}\right) \cdots,
\end{array}
$$

obtaining $a_{15234}+a_{51234}+a_{15243}+a_{51243}=0$. By the induction hypothesis $a_{51234}+\alpha_{51243}=0$, hence $\alpha_{15234}+\alpha_{15243}=0$, as required. Case (a) is now settled when $x_{3}$ and $x_{4}$ have distance zero between them. We proceed by induction on that distance, assuming it is now greater than zero. The monomial has the form $M_{\sigma}=\cdots x_{1} \cdots x_{2} \cdots x_{3} x_{5} \cdots x_{4} \cdots$, so by the induction hypothesis and what has already been proved, we may write $\alpha_{12354}=-\alpha_{12534}=\alpha_{12543}=$ $-a_{12453^{\circ}}$. This completes the proof of case (a).

(b) The pair $x_{1}, x_{2}$ separates the pair $x_{3}, x_{4}$ in the form, say, $M_{\sigma}=\cdots x_{3}$ $\cdots x_{1} \cdots x_{2} \cdots x_{4} \cdots$. Suppose first the number of variables between $x_{3}$ and $x_{4}$ is odd. Theorem 5 may then be applied to produce a semirigid substitution of the form

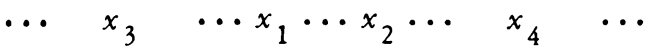

$$
\begin{aligned}
& \ldots\left(e_{11}\right) \quad \ldots \quad\left(e_{11}\right) \ldots
\end{aligned}
$$

Denoting by [12] the whole block of indices between $x_{3}$ and $x_{4}$, we have $\left(a_{3[12] 4}+a_{4[12] 3}\right)+\left(a_{34[12]}+a_{43[12]}\right)+\left(a_{[12] 34}+\alpha_{[12] 43}\right)=0$. The second and third parenthesized terms vanish by (a), leaving us with $\alpha_{3124}+\alpha_{4123}=0$ 
as required. If the number of variables between $x_{3}$ and $x_{4}$ is even, it is certainly not $2 n-1$, so some variable must occur in $M_{\sigma}$ outside the block $x_{3} \cdots x_{4}$ : We may suppose then that our monomial is of the form $M_{\sigma}=\cdots x_{3} \cdots x_{1} \cdots$ $x_{2} \cdots x_{4} x_{5} \cdots$ Using case (a) and the part of (b) already proved, we have $\alpha_{31245}=-a_{31254}=\alpha_{41253}=-\alpha_{41235}$, as required.

(c) The pair $x_{3}, x_{4}$ separates the pair $x_{1}, x_{2}$, say $M_{\sigma}=\cdots x_{1} \cdots x_{3} \cdots$ $x_{4} \cdots x_{2} \cdots$. It will suffice to treat the case where $x_{4}$ immediately succeeds $x_{3}$, for in any monomial of type (c) we may transpose $x_{3}$ and $x_{4}$ by successively transposing immediate neighbors, none of which involves an immediate neighbor of $x_{1}$ or $x_{2}$. The number of the successive transpositions in this process must be odd, hence if the conclusion holds for them it will hold for the transposition (3 4). If the number of variables between $x_{1}$ and $x_{2}$ is odd, we substitute

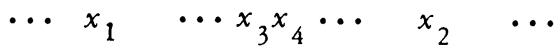

$$
\begin{aligned}
& \cdots\left(e_{11}\right) \quad \ldots \quad\left(e_{11}\right) \quad \ldots
\end{aligned}
$$

and obtain (by (12)-symmetry) $a_{1[34] 2}+\alpha_{12[34]}+\alpha_{[34] 12}=0$. We now interchange in this substitution the values assigned to $x_{4}$ and $x_{5}$, thus obtaining $a_{1[43] 2}+a_{12[43]}+a_{12[43\}}=0$. Adding the two equalities and applying case (a), we arrive at the required relation $\alpha_{1[34] 2}+\alpha_{1[43]_{2}}=0$. Next assume the number of variables between $x_{1}$ and $x_{2}$ is even. Then the monomial has the form $M_{\sigma}=\cdots x_{1} \cdots x_{3} x_{4} \cdots x_{2} x_{5} \cdots$, and we substitute

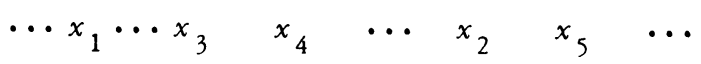

$$
\begin{aligned}
& \begin{array}{lllllll}
\cdots & e_{11} & \left(e_{11}\right) & \cdots & e_{22} & \left(e_{22}\right) & \ldots
\end{array}
\end{aligned}
$$

obtaining $\alpha_{13425}+\alpha_{14325}+\alpha_{13452}+\alpha_{14352}=0$. But $\alpha_{13452}+\alpha_{14352}=0$ by the odd case, hence $\alpha_{13425}+\alpha_{14325}=0$.

(d) The two pairs are "interlaced", e.g. $M_{\sigma}=\cdots x_{3} \cdots x_{1} \cdots x_{4} \cdots x_{2} \cdots$ If the number of variables between $x_{3}$ and $x_{4}$ is odd we substitute

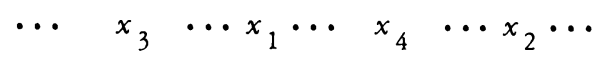

$$
\begin{aligned}
& \ldots\left(e_{11}\right) \quad \ldots \quad\left(e_{11}\right) \quad \ldots,
\end{aligned}
$$

obtaining as before a reduction to cases (a) and (c). Suppose then there is an even number of variables between $x_{3}$ and $x_{4} \cdot x_{1}$ and $x_{3}$ are separated, by assumption, by at least one variable and so are $x_{1}$ and $x_{4}$, but at least one of these pairs must be separated by more than one variable. For otherwise $x_{3}$ and $x_{4}$ would be separated by 3 variables which is impossible. Thus the monomial may be assumed to have the form $M_{\sigma}=\cdots x_{3} x_{5} x_{6} \cdots x_{1} \cdots x_{4} \cdots x_{2} \cdots$. By case (a) and the part of (d) we have already proved we have (notice that $x_{5}$ is not an 
immediate neighbor of $x_{1}$ ): $a_{35142}=-a_{53142}=a_{54132}=-a_{45132}$. This concludes case (d), thereby completing the proof of Lemma 12.

Definition. The pair $\left(x_{i_{1}}, x_{i_{2}}\right)$ touches the pair $\left(x_{j_{1}}, x_{j_{2}}\right)$ in the monomial $M_{\sigma}$ if one of the first pair is immediate neighbor of one of the second.

Corollary 13. Let $f \in A$ be a 2-symmetric polynomial and let $M_{\sigma}$ be a monomial of $f$ in which the pair $\left(x_{1}, x_{2}\right)$ does not touch the pair $\left(x_{3}, x_{4}\right)$. Then $\alpha_{\sigma}=0$.

Proof. Since $f$ is $(3,4)$-symmetric, $\alpha_{(34) \sigma}=a_{\sigma}$. On the other hand, $\alpha_{(34) \sigma}$ $=-a_{\sigma}$ by Lemma 12. Thus $a_{\sigma}=-a_{\sigma}$, i.e. $a_{\sigma}=0$.

Lemma 14. If $f \in A$ is 3-symmetric then $f=0$.

Proof. $f$ is symmetric in 3 disjoint pairs of variables, say $\left(x_{1}, x_{2}\right),\left(y_{1}, y_{2}\right)$ and $\left(z_{1}, z_{2}\right)$. Suppose $f \neq 0$, i.e. some monomial $M_{\sigma}$ occurs in $f$ with coefficient $a_{\sigma} \neq 0$. By Lemma 12 we may pass from $M_{\sigma}$ to another monomial $M_{\sigma}$ of $f$ by transposing a pair of variables not touching one of the symmetric pairs, and still have $a_{\sigma^{\prime}} \neq 0$. Also by Corollary 13, any two of the symmetric pairs must touch in $M_{\sigma}$, and in any other monomial with nonzero coefficient. Let $u$ be a new variable (i.e. not one of $x_{i}, y_{i}$ or $z_{i}$ ), which must occur in $M_{\sigma}$ since the total number $2 n+1$ of variables is odd. For the rest of the proof we disregard all variables except the above mentioned seven, and the terms "variable" or "immediate neighbor" are correspondingly restricted. If the pairs $\left(x_{1}, x_{2}\right)$ and $\left(y_{1}, y_{2}\right)$ do not touch, we shall say there is no $(x, y)$-contact. Of course if there is no $(x, y)$ contact in the restricted sense, the same is true when all variables are considered. Now to the proof. We distinguish several cases by the relative position of the variable $u$ in the monomial $M_{\sigma}$. In each case we shall show that by "admissible" permutations (i.e. such permutations as do not annihilate the monomial's coefficient) one can pass from $M_{\sigma}$ to an $M_{\sigma^{\prime}}$, in which two of the symmetric pairs do not touch, contradicting Corollary 13.

(a) All six variables are on one side, say left, of $u$. One of the three symmetric pairs contains none of $u$ 's two nearest neighbors on the left. Thus $u$ and its nearest neighbor on the left do not touch this symmetric pair, and they can be transposed without annihilating the coefficient of the monomial. But this leads to a contradiction by case (b).

(b) A single variable is on one side of $u$ and the rest on the other side. We may assume that $x_{1}$ is on the right, and the rest on the left of $u$. In order that all symmetric pairs should touch each other, $x_{2}$ must occur between $y_{i}$ and $z_{j}$, say in the form $y_{1} x_{2} z_{1}$. Let us now locate $y_{2}$ and $z_{2}$ in the monomial, noting first that they do not touch $\left(x_{1}, x_{2}\right)$ and thus may be interchanged. Since the 
block $y_{2} y_{1} x_{2} z_{1} z_{2}$ cannot occur in $M_{\sigma}$ (no $(y, z)$-contact), it follows that also $z_{2} y_{1} x_{2} z_{1} y_{2}$ cannot occur. Thus, by suitably interchanging $y_{2}$ and $z_{2}$ (if necessary) we may assume that $M_{\sigma}$ has one of the following forms: either $M_{\sigma}=$ $y_{1} x_{2} z_{1} z_{2} y_{2} u x_{1}$ or $M_{\sigma}=z_{2} y_{2} y_{1} x_{2} z_{1} u x_{1}$. In the former case we may interchange $x_{1}$ and $y_{1}$ (they do not touch $\left(z_{1}, z_{2}\right)$ ), concluding that the monomial $x_{1} x_{2} z_{1} z_{2} y_{2} u y_{1}$ has nonzero coefficient. But this is impossible since there is no $(x, y)$-contact in this monomial. In the latter case we may interchange $x_{1}$ and $z_{1}$ (they do not touch $\left(y_{1}, y_{2}\right)$ ), obtaining the monomial $z_{2} y_{2} y_{1} x_{2} x_{1} u z_{1}$ in which there is no $(x, z)$-contact-a contradiction again.

(c) Two variables on one side of $u$ and four on the other. The two could not belong to the same symmetric pair, for this pair then would not touch the other symmetric pairs. Thus we may suppose that $M_{\sigma}=\cdots u x_{1} y_{1}$. If $u$ 's immediate neighbor on the left is not $z_{i}$, then $u$ and $x_{1}$ do not touch $\left(z_{1}, z_{2}\right)$ and can be interchanged, leading to case (b). We may therefore suppose that $M_{\sigma}=\cdots$ $z_{1} u x_{1} y_{1} \cdot z_{1}$ 's immediate neighbor on the left is not $z_{2}$, for then the pair $\left(z_{1}, z_{2}\right)$ would have only one contact with the other pairs. It may be supposed that this neighbor is $y_{2}$, for otherwise we can interchange $x_{1}$ and $y_{1}$ on the right of $u$ and treat similarly. Thus $M_{\sigma}=\cdots y_{2} z_{1} u x_{1} y_{1}$. The leftmost variable in $M_{\sigma}$ does not touch $y_{2}$, for there are at least 7 variables, and so may be interchanged with $u$ (they do not touch $\left(y_{1}, y_{2}\right)$ ). But this leads us back to case (a).

(d) Three variables on each side of $u$. The two members of a symmetric pair do not occur on the same side of $u$, for then they would have only one contact with the other pairs. Assume $M_{\sigma}=\cdots u x_{1} y_{1} z_{1}$. If $u$ 's immediate neighbor on the left is not $z_{2}$, we may interchange $u$ and $x_{1}$ (no $z$-contact) and get back to case (c). So assume $M_{\sigma}=\cdots z_{2} u x_{1} y_{1} z_{1}$. For an $(x, z)$-contact to exist it is necessary that $M_{\sigma}=y_{2} x_{2} z_{2} u x_{1} y_{1} z_{1}$. But here $x_{1}$ and $y_{2}$ may be interchanged (no $z$-contact) to get the monomial $x_{1} x_{2} z_{2} u y_{2} y_{1} z_{1}$ with no $(x, y)$-contact. be zero.

We have shown that all the possibilities lead to a contradiction, so $f$ must

Corollary 15. Let $f \in A$ be 2-symmetric. Then $f$ is skew-symmetric in any pair of variables distinct from $x_{1}, x_{2}, x_{3}, x_{4}$, that is, $f$ is 2-perfect.

Proof. Let there be given such a pair, say $\left(x_{5}, x_{6}\right)$. Then $\sigma_{56} f$ still belongs to $A$ and is 3-symmetric. Thus by Lemma $14, \sigma_{56} f=0$, i.e. $f$ is skewsymmetric in $\left(x_{5}, x_{6}\right)$.

Definition. $f \in A$ will be called symmetric in 3 variables $\left(x_{1}, x_{2}, x_{3}\right)$ if $f$ is invariant under any permutation of $\{1,2,3\}$. Equivalently, $f$ is symmetric in $\left(x_{1}, x_{2}, x_{3}\right)$ if it is (1 2)- and (2 3)-symmetric. 
Corollary 16. Let $f \in A$ be an identity of $F_{n}, n>2$, symmetric in $\left(x_{1}, x_{2}\right)$ and $\left(x_{3}, x_{4}, x_{5}\right)$. Then $f=0$.

Proof. Since $n>2,2 n+1 \geq 7$ so there is an additional variable $x_{6}$. We are given (1 2) $f=\left(\begin{array}{l}34 \\ 4\end{array}\right) f=\left(\begin{array}{l}45 \\ 5\end{array}\right) f=\left(\begin{array}{l}3 \\ 5\end{array}\right) f=f$. By Corollary $15,(56) f=-f$ since

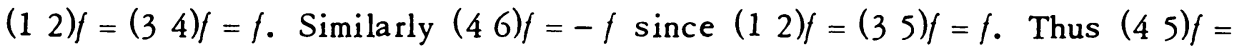
$(56)(46)(56) f=(-1)^{3} f=-f$. But $(45) f=f$ so $f=-f$ and $f=0$.

Remark. To see that the restriction $n>2$ in Corollary 16 is essential, start with the identity $[[x y][u v]+[u v][x y], z]$ of $F_{2}$ and symmetrize in $(x, u)$ and in $(y, v, z)$ to get a nonzero identity satisfying the assumptions of the corollary.

Corollary 17. If $f \in A$ is 2-symmetric, then $g=f+(45) f+(35) f=0$.

Proof. Clearly $\left(\begin{array}{ll}1 & 2\end{array}\right) g=g$ since $\left(\begin{array}{ll}1 & 2\end{array}\right) f=f$. We claim that $(35) g=(45) g=g$, so by Corollary 16, $g=0$. Indeed, $(35) g=(35) f+(35)(45) f+(35)^{2} f=(35) f+$ $(45)(34) f+f=(35) f+(45) f+f=g$. Similarly, $(45) g=g$.

We are now ready to prove the 2-symmetric analogue of Lemma 10.

Lemma 18. Let $f \in A$ be 2-symmetric identity of $F_{n}, n>2$, in which the monomials $x_{1} x_{2} x_{3} x_{4} x_{5} \cdots x_{2 n+1}$ and $x_{4} x_{3} x_{2} x_{1} x_{5} \cdots x_{2 n+1}$ occur with zero coefficients. Then $f=0$.

Proof. Assume $f \neq 0$, so some monomial $M_{\sigma}$ occurs with coefficient $\alpha_{\sigma} \neq 0$. We split the proof into two steps.

Step (a). We claim that a monomial with nonzero coefficient exists in $f$, in which there is a single contact between $\left(x_{1}, x_{2}\right)$ and $\left(x_{3}, x_{4}\right)$. Indeed, the monomial $M_{\sigma}$ we started with must have a certain (positive) number of contacts between $\left(x_{1}, x_{2}\right)$ and $\left(x_{3}, x_{4}\right)$, or it would have zero coefficient by Corollary 13. If the number of these contacts is 1 , there is nothing to prove. Suppose now there are two such contacts. Then in one of the pairs both members must take part in the contacts. Assume this pair is $\left(x_{1}, x_{2}\right)$. (The two contacts may occur in various ways, such as $x_{1} x_{3} x_{2}, x_{1} x_{3} \ldots: x_{2} x_{4}, x_{1} x_{3} x_{4} x_{2}$ etc.) We claim that an additional variable must occur in $M_{\sigma}$, which does not touch the pair $\left(x_{3}, x_{4}\right)$. Indeed, $x_{3}$ and $x_{4}$ have 4 sides and so at most 4 contacts with other variables, and two of these contacts are already occupied by $x_{1}$ and $x_{2}$. Thus $x_{3}$ and $x_{4}$ have at most 2 contacts with variables other than $x_{1}$ and $x_{2}$. But there are in $M_{\sigma}$ at least 3 variables other than $x_{1}, x_{2}, x_{3}, x_{4}$ (for $2 n+1 \geq 7$ ), so one of them, say $x_{5}$, does not touch $x_{3}$ and $x_{4}$. By Corollary $17 g=f+(15) f+(25) f$ $=0$. Equating to zero the coefficient of $M_{\sigma}$ in $g$ we get $\alpha_{\sigma}+\alpha_{(15) \sigma}+\alpha_{(25) \sigma}=$ 0 . As $a_{\sigma} \neq 0$, one of the other terms, say $\alpha_{(15) \sigma}$ is also nonzero. But $\alpha_{(15) \sigma}$ is the coefficient in $f$ of a monomial obtained from $M_{\sigma}$ by interchanging $x_{1}$ and $x_{5}$, thus having only one contact between $\left(x_{1}, x_{2}\right)$ and $\left(x_{3}, x_{4}\right)$. Suppose finally 
that $M_{\sigma}$ has more than two contacts between $\left(x_{1}, x_{2}\right)$ and $\left(x_{3}, x_{4}\right)$. Then one variable, say $x_{2}$, must touch both members of the other pair and $M_{\sigma}$ contains a block of the form $x_{3} x_{2} x_{4}$. In order that there should be more than two contacts, $x_{1}$ must touch this block, so we may assume $M_{\sigma}=\cdots x_{1} x_{3} x_{2}{ }^{x}{ }_{4} \cdots$ : Substitute in $f$ a semirigid substitution of the form

$$
\begin{array}{ccccc}
\cdots & x_{1} & x_{3} & x_{2} & \cdots \\
\cdots & e_{11} & \left(e_{11}\right) & \left(e_{11}\right) & \cdots
\end{array}
$$

and obtain the equation $\alpha_{1324}+a_{1234}+a_{3124}=0$. Since the first term dœs not vanish (this is $a_{\sigma}$ ), neither does one of the other terms. The second term is the coefficient of a monomial with only one contact, and the third, with two contacts. Therefore this case is reduced to one of the former cases. For the rest of the proof we assume, then, that in $M_{\sigma}$ there is a single contact between $\left(x_{1}, x_{2}\right)$ and $\left(x_{3}, x_{4}\right)$.

Step (b). Interchanging $x_{1}, x_{2}$ or $x_{3}, x_{4}$ does not change the coefficients of monomials of $f$. Therefore we may deduce from step (a) that a monomial with nonzero coefficient exists in $f$, in which the single contact between $\left(x_{1}, x_{2}\right)$ and $\left(x_{3}, x_{4}\right)$ is $x_{2} \cdot x_{3}$ or $x_{3} \cdot x_{2}$. Suppose for instance that in $M_{\sigma}$ the contact is $x_{2} \cdot x_{3}$. We shall then show that the coefficient of $x_{1} x_{2} x_{3} \cdot: x_{2 n+1}$ is nonzero, contradicting the assumptions. (The case of contact $x_{3} \cdot x_{2}$ leads similarly to the conclusion that $\alpha_{43215 \ldots ; n_{n+1}} \neq 0$, which is also a contradiction.) By Corollary 15 , permuting the variables $x_{5}, \cdots, x_{2 n+1}$ does not influence the vanishing or nonvanishing of the coefficient, so no importance will be attached to their order in the sequel. We distinguish several cases according to the relative order of $x, x_{2} \cdot x_{3}, x_{4}$ in the monomial.

Case (0). $M_{\sigma}$ contains the block $x_{1} x_{2} x_{3} x_{4}$. We shall show that this block can always be shifted one position to the left without annihilating the coefficient. Thus by a finite number of shifts the contradiction is obtained. If there are at least two more variables on the left of the block, $M_{\sigma}$ has the form $\cdots: x_{5} x_{6} x_{1} x_{2} x^{x} x_{4}$ and we make the following (semirigid) substitution:

$$
\begin{array}{cccccccc}
\cdots & x_{5} & x_{6} & x_{1} & x_{2} & x_{3} & x_{4} & \cdots \\
\cdots & \left(e_{11}\right) & e_{12} & e_{22} & \left(e_{22}\right) & e_{21} & e_{13} & \cdots
\end{array}
$$

By the (12)-symmetry we get as usual, $\alpha_{561234}+\alpha_{612354}=0$, so $\alpha_{612354} \neq 0$. Since $x_{4}, x_{5}$ do not touch $\left(x_{1}, x_{2}\right)$, we can interchange them to get the required conclusion $\alpha_{612345} \neq 0$. If there are less than two variables on the left of the block then either there are none, in which case we have nothing to prove, or there is only one. In this case, since $M_{\sigma}$ has at least 7 variables, there must be at 
least two variables on the right of the block, so that $M_{\sigma}$ has the form $x_{5} x_{1} x_{2} x_{3} x_{4} x_{6} x_{7}$. Here $x_{5}$ and $x_{1}$ do not touch $\left(x_{3}, x_{4}\right)$ so we conclude that also $a_{1523467} \neq 0$. We substitute

$$
\begin{array}{cccccccc}
x_{1} & x_{5} & x_{2} & x_{3} & x_{4} & x_{6} & x_{7} & \cdots \\
& \left(e_{11}\right) & e_{12} & e_{22} & \left(e_{22}\right) & e_{21} & & \cdots,
\end{array}
$$

and get (using (34)-symmetry) $\alpha_{1523467}+\alpha_{1234657}=0$. This implies $\alpha_{1234657}$ $\neq 0$, concluding case $(0)$.

Case (1). $M_{\sigma}=\cdots x_{1} \cdots: x_{2} x_{3} \cdots x_{4} \cdots$ By Lemma 12 interchange $x_{1}$ with $x_{2}$ 's immediate neighbor on the left and $x_{4}$ with $x_{3}$ 's immediate neighbor on the right, getting a contradiction by case (0).

Case (2). $M_{\sigma}=\cdots x_{4} \cdots x_{2} x_{3} \cdots x_{1} \cdots$ : By Lemma 12 interchange $x_{4}$ with the leftmost variable in $M_{\sigma}$ and $x_{1}$ with the rightmost. The following monomial has therefore nonzero coefficient: $x_{4} \cdots x_{2} x_{3} \cdots x_{1}$. Since there are at least 7 variables, either $x_{4}$ and $x_{2}$, or $x_{3}$ and $x_{1}$ are separated by at least two variables, and we may assume that the monomial has the form $x_{4} \cdots x_{2} x_{3} x_{5} x_{6}$ $\cdots x_{1}$. Since $x_{4}$ and $x_{5}$ do not touch $\left(x_{1}, x_{2}\right)$ we may pass to $x_{5} \cdots x_{2} x_{3} x_{4} x_{6}$ $\cdots x_{1}$, hence to $x_{5} \cdots x_{1} x_{2} x_{3} x_{4} x_{6} \cdots$, thus obtaining a reduction to case $(0)$.

Case (3). $M_{\sigma}=\cdots x_{2} x_{3} \cdots x_{4} \cdots x_{1} \cdots$, from which we pass to $\cdots x_{2} x_{3} x_{4}$ $\cdots x_{1} \cdots$ If $x_{2}$ is not in the leftmost position we can pass to $\cdots x_{1} x_{2} x_{3} x_{4}$ $\cdots$, which is again case (0). If $x_{2}$ is in the leftmost position, substitute

$$
\begin{array}{cccccc}
x_{2} & x_{3} & x_{4} & \cdots & x_{1} & \cdots \\
e_{12} & e_{22} & \left(e_{22}\right) & \cdots & \left(e_{11}\right) & \cdots
\end{array}
$$

and conclude $\alpha_{1234 \ldots} \ldots 0$, a contradiction.

Case (4). $M_{\sigma}=\cdots x_{2} x_{3} \cdots x_{1} \cdots x_{4} \cdots$, from which we pass to $\cdots x_{2} x_{3}$ $\cdots x_{1} \cdots x_{4}$. If $x_{2}$ is not in the leftmost position, pass to $\cdots x_{1} x_{2} x_{3} \cdots x_{4}$, hence to case (1). If $x_{2}$ is in the leftmost position interchange $x_{1}$ with the variable in the $(2 n-1)$ th position. Since there are at least 7 variables, the monomial thus obtained has the form $x_{2} x_{3} x_{6} x_{7} \cdots x_{1} x_{5} x_{4}$, and interchanging $x_{4}$ and $x_{6}$ (they do not touch $\left(x_{1}, x_{2}\right)$ ) we arrive at the monomial $x_{2} x_{3} x_{4} x_{7} \cdots$ $x_{1} x_{5} x_{6}$ of case (3).

The remaining cases, $M_{\sigma}=\cdots x_{4} \cdots x_{1} \cdots x_{2} x_{3} \cdots$ and $M_{\sigma}=\cdots x_{1} \cdots$ $x_{4} \cdots x_{2} x_{3} \cdots$, are treated exactly as cases (3) and (4) respectively, interchanging left and right.

Corollary 19. If $n>2$, then every 2-symmetric polynomial in $A$ belongs to $B$.

Proof. Let $f \in A$ be 2-symmetric and denote by $\alpha_{1}$ and $\alpha_{2}$ the coefficients of $x_{1} x_{2} x_{3} x_{4} x_{5} \cdots x_{2 n+1}$ and $x_{4} x_{3} x_{2} x_{1} x_{5} \cdots x_{2 n+1}$ in $f$. Define $g=\alpha_{2} q_{2}-a_{1} q_{1}$ (see the 
notation preceding Lemma 12), and note that $g$ belongs to $B$ and has the same coefficients as $f$ for the above monomials. Therefore $f-g \in A$ is a 2 -symmetric polynomial for which these coefficients vanish, so by Lemma $18 f-g=0$, i.e. $f=g \in B$.

Combining Theorem 7, Theorem 11 and Corollary 19 we arrive at the main theorem.

Theorem 20. If $n>2$, then $B=A$, i.e. every multilinear identity of $F_{n}$ of degree $2 n+1$ follows from the standard identity $s_{\eta_{n}}$.

Remark. Theorem 20 is false for $n=2$, for it can be show $n$ that the identity $\left[\left[x_{1} x_{2}\right]\left[x_{3} x_{4}\right]+\left[x_{3} x_{4}\right]\left[x_{1} x_{2}\right], x_{5}\right]$ does not follow from $s_{4}$.

5. The dimension of the space of identities $A$. In this section we compute the dimension of the space of identities $A$, assuming $n>2$. By Theorem 20 we know that $A$ is spanned by the identities $\phi_{i}, \psi_{i}, \chi_{i j}$ (defined at the beginning of $\$ 3)$. Later on we shall show that the $\phi_{i}^{\prime}$ 's and the $\chi_{i j}$ 's excluding $\chi_{12}$, form a basis for $A$, hence $\operatorname{dim} A=(2 n+1)^{2}-1$. Consider first the operation of $\sigma_{k k+1}(=1 / 2(1+(k k+1)))$ on any poly nomial spanned by the $\phi_{i}$ 's and $\chi_{i j}$ 's. Let

$$
f=\sum_{i=1}^{2 n+1} \alpha_{i} \phi_{i}+\sum_{i, j=1 ; i \neq j}^{2 n+1} \gamma_{i j} \chi_{i j}
$$

and apply $\sigma_{k k+1}$ to $f$. If $i, j \neq k, k+1$ then $\sigma_{k k+1} \phi_{i}=\sigma_{k k+1} \chi_{i j}=0$, therefore

$$
\begin{aligned}
\sigma_{k k+1} f= & \sigma_{k k+1}\left[\alpha_{k} \phi_{k}+\alpha_{k+1} \phi_{k+1}+\sum_{i=1 ; i \neq k, k+1}^{2 n+1}\left(\gamma_{i k} \chi_{i k}+\gamma_{i k+1} x_{i k+1}\right)\right. \\
& \left.+\sum_{j=1 ; j \neq k, k+1}^{2 n+1}\left(\gamma_{k j} \chi_{k j}+\gamma_{k+1 j} \chi_{k+1 j}\right)+\left(\gamma_{k k+1} x_{k k+1}+\gamma_{k+1 k} \chi_{k+1 k}\right)\right]
\end{aligned}
$$

Using the list preceding Lemma 8 , we have

$$
\begin{aligned}
2 \sigma_{k k+1} f= & \alpha_{k}\left(\phi_{k}+\phi_{k+1}\right)+\alpha_{k+1}\left(\phi_{k}+\phi_{k+1}\right) \\
& +\sum_{i \neq k, k+1}\left(\gamma_{i k}\left(\chi_{i k}+\chi_{i k+1}\right)+\gamma_{i k+1}\left(\chi_{i k+1}+\chi_{i k}\right)\right) \\
& +\sum_{j \neq k, k+1}\left(\gamma_{k j}\left(\chi_{k j}-\chi_{k+1 j}\right)+\gamma_{k+1 j}\left(\chi_{k+1 j}-\chi_{k j}\right)\right) \\
& +\gamma_{k k+1}\left(\chi_{k k+1}+\chi_{k+1 k}\right)+\gamma_{k+1 k}\left(\chi_{k+1 k}+\chi_{k k+1}\right) \\
= & \left(a_{k}+a_{k+1}\right)\left(\phi_{k}+\phi_{k+1}\right)+\sum_{i \neq k, k+1}\left(\gamma_{i k}+\gamma_{i k+1}\right)\left(\chi_{i k}+\chi_{i k+1}\right) \\
& +\sum_{j \neq k, k+1}\left(\gamma_{k j}-\gamma_{k+1 j}\right)\left(\chi_{k j}-\chi_{k+1 j}\right)+\left(\gamma_{k k+1}+\gamma_{k+1 k}\right)\left(\chi_{k k+1}+\chi_{k+1 k}\right) .
\end{aligned}
$$


The next lemma shows that the $(k k+1)$-symmetric terms of the last expression are linearly independent.

Lemma 21. Let

$$
\begin{aligned}
g= & a\left(\phi_{k}+\phi_{k+1}\right)+\sum_{i=1 ; i \neq k, k+1}^{2 n+1} c_{i}\left(\chi_{k i}-\chi_{k+1 i}\right) \\
& +\sum_{i=1 ; i \neq k, k+1}^{2 n+1} d_{i}\left(\chi_{i k}+\chi_{i k+1}\right)+e\left(\chi_{k k+1}+\chi_{k+1 k}\right) .
\end{aligned}
$$

If $g=0$ then $a=c_{i}=d_{i}=e=0$ for $i=1, \cdots, k-1, k+2, \cdots, 2 n+1$.

Proof. In this proof, $\hat{x}_{k}$ will mean " $x_{k}$ omitted" and $\pm b$ will mean "plus or minus $b . "$ In the equation $g=0$, equate to zero the coefficients of the monomials $x_{1} \cdots \hat{x}_{k} \hat{x}_{k+1} \cdots x_{2 n+1} x_{k} x_{k+1}$ and $x_{1} \cdots \hat{x}_{k} \hat{x}_{k+1} \cdots \hat{x}_{i} \cdots x_{2 n+1} x_{k} x_{k+1} x_{i}$ to obtain, respectively, the equations $\pm\left(d_{2 n+1}+e\right)=0$ and $\pm\left(d_{2 n+1}+e \pm c_{i}\right)=0$. It follows that $c_{i}=0$ for $i=1, \cdots, k-1, k+2, \cdots, 2 n+1$. Similarly, the monomial $x_{1} \cdots x_{k-1} x_{k} x_{i} x_{k+1} \cdots \hat{x}_{i} \cdots x_{2 n+1}(i \neq k-1, k, k+1)$ leads to $\pm\left(d_{k-1}-d_{i}\right)=0$, or $d_{i}=d_{k-1}$, i.e. all the $d^{\prime}$ 's are equal. (We assume here $k>$ 1. For $k=1$ we get the same result from $x_{2 n+1} x_{1} x_{i} x_{2} \cdots \hat{x}_{i} \cdots x_{2 n}$. This remark applies also in the next two cases.). The monomial $x_{k} x_{1} \cdots x_{k-1} x_{k+1} \cdots$ $x_{2 n+1}$ yields $a+(-1)^{k-1} d_{k-1}=0$, but if we interchange the block $x_{k-1} x_{k+1}$ in this monomial with its neighbor on the right (or on the left, if $k+1$ happened to be $2 n+1)$ we get $a+(-1)^{k} d_{k-1}=0$. Thus $a=d_{k-1}=0$ and since all the $d_{i}$ 's are equal, they all vanish. From the first equation $d_{2 n+1}+e=0$ we finally conclude that $e=0$.

In particular, we shall require the following

Corollary 22. The following polynomials are linearly independent:

$$
\begin{array}{ll}
p_{1}=\phi_{1}+\phi_{2}, & p_{3}=\chi_{12}+\chi_{21}, \\
p_{4}=\sum_{i=3}^{2 n+1}\left(\chi_{i 1}+\chi_{i 2}\right), & p_{5}=\sum_{i=3}^{2 n+1}(-1)^{i}\left(\chi_{1 i}-\chi_{2 i}\right) .
\end{array}
$$

(Compare Lemma 9.)

We are now ready to construct a basis for $A$.

Lemma 23. The polynomials $\phi_{i}, i=1, \cdots, 2 n+1$, and $\chi_{i j}, i, j=1, \cdots$, $2 n+1, i \neq j,(i j) \neq 1(12)$, span $A$.

Proof. We shall first show, using Theorem 7, that the $\phi_{i}$ 's together with all 
of the $\chi_{i j}$ 's span $A$. Indeed, the standard polynomial is spanned by the $\phi_{i}$ 's, for $s_{2 n+1}=\phi_{1}-\phi_{2}+\cdots+\phi_{2 n+1}$. Also, it is clear that the 2-symmetric polynomials in $B$ (hence in $A$ ) are spanned by the $\chi_{i j}$ 's. It will therefore suffice to treat the 1-perfect polynomials and we do this, say, for the (1 2 )-perfect case. In $\$ 3$ it was shown the dimension of the space of the (12)-perfect polynomials is 4. The polynomials $p_{1}, p_{3}, p_{4}, p_{5}$ occurring in L.emma 22 are (1 2)-perfect (compare Lemma 9), and being independent they form a basis for that space. But they are clearly spanned by the $\phi_{i}$ 's and $\chi_{i j}$ 's.

Next we claim that the polynomial $b=\sum_{j=1}^{2 n+1}(-1)^{j} \sum_{i=1 ; i \neq j}^{2 n+1} \chi_{i j}$ is the zero polynomial. Once the claim is established, it is clear that $\chi_{12}$ is spanned by the rest of the $\chi_{i j}$ 's and the proof of the lemma will be completed. For a fixed $j$, consider the polynomial $\sum_{i=1 ; i \neq j}^{2 n+1} \chi_{i j}$. This polynomial is (kl)-skew symmetric for all $k, l \neq j$, for by Lemma 8 we have

$$
\sigma_{k l}\left(\sum_{i=1 ; i \neq j}^{2 n+1} \chi_{i j}\right)=\sigma_{k l}\left(\chi_{k j}+\chi_{l j}\right)=0 .
$$

Now consider $\sigma_{k l} h$. By what we have just seen, $\sigma_{k l}$ annihilates all the terms in the summation on $j$, except possibly those corresponding to $j=k$ and $j=l$. Thus

$$
\begin{aligned}
\sigma_{k l} b & =\sigma_{k l}\left[(-1)^{k} \sum_{i=1 ; i \neq k}^{2 n+1} \chi_{i k}+(-1)^{l} \sum_{i=1 ; i \neq l}^{2 n+1} X_{i l}\right] \\
& =(-1)^{k}\left[\sigma_{k l}\left(\chi_{l k}+(-1)^{l-k} \chi_{k l}\right)+\sum_{i=1 ; i \neq k, l}^{2 n+1} \sigma_{k l}\left(\chi_{i k}+(-1)^{l-k} \chi_{i l}\right)\right],
\end{aligned}
$$

and we conclude by Lemma 8 that $\sigma_{k, l} b=0$. Since $b$ is skew symmetric in every pair of variables, $b=a s_{2 n+1}$ for some $a \in F$. But the coefficient of $x_{1} x_{2} \cdots$ $x_{2 n+1}$ in $b$ is 0 (to see this denote the coefficient of $\chi_{i j}$ in the sum defining $b$ by $a_{i j}$ and note that the coefficient of $x_{1} \cdots x_{2 n+1}$ in $b$ is $a_{12}+a_{23}+$ $\left.a_{34}+\cdots+a_{2 n 2 n+1}=(1-1)+(1-1)+\cdots+(1-1)=0\right)$, so $a=0$ and $b=0$.

Theorem 24. The polynomials $\phi_{i}, i=1, \cdots, 2 n+1$ and $\chi_{i j}, i, j=1, \cdots$, $2 n+1, i \neq j,(i, j) \neq\left(\begin{array}{ll}1 & 2\end{array}\right)$ form a basis for $A$.

Proof. With Lemma 23 in our hands, we have only to prove that the polynomials are linearly independent. Let $f=\sum_{i=1}^{2 n+1} \alpha_{i} \phi_{i}+\sum_{i, j=1 ; i \neq j}^{2 n+1} \gamma_{i j} \chi_{i j}$, where $\gamma_{12}=0$ is put in for corvenience, and assume $f=0$. By the computations preceding Lemma 21 we can write 


$$
\begin{aligned}
2 \sigma_{k k+1} f= & \left(\alpha_{k}+\alpha_{k+1}\right)\left(\phi_{k}+\phi_{k+1}\right)+\sum_{i=1 ; i \neq k, k+1}^{2 n+1}\left(\gamma_{i k}+\gamma_{i k+1}\right)\left(\chi_{i k}+\chi_{i k+1}\right) \\
& +\sum_{j=1 ; j \neq k, k+1}^{2 n+1}\left(\gamma_{k j}-\gamma_{k+1 j}\right)\left(\chi_{k j}-\chi_{k+1 j}\right) \\
& +\left(\gamma_{k k+1}+\gamma_{k+1 k}\right)\left(\chi_{k k+1}+\chi_{k+1 k}\right)=0 .
\end{aligned}
$$

It follows from Lemma 21 that, for all $i, j \neq k, k+1, \gamma_{i k}+\gamma_{i k+1}=\gamma_{k j}-\gamma_{k+1 j}=0$, that is, $\gamma_{i k}=-\gamma_{i k+1}$ and $\gamma_{k j}=\gamma_{k+1 j}$. These relations clearly imply that, for all $r, s, \gamma_{r s}= \pm \gamma_{12}=0$. The given equality $f=0$ now reduces to $\Sigma \alpha_{i} \phi_{i}=0$, from which it easily follows that $\alpha_{i}=0$ for $i=1, \cdots, 2 n+1$ (e.g. consider the coefficient of $\left.x_{i} x_{1} \cdots \hat{x}_{i} \cdots x_{\cdot 2 n+1}\right)$.

Corollary 25. $\operatorname{dim} A=(2 n+1)^{2}-1=4 n(n+1)$.

6. An example. In this section we introduce an identity in $A$, which is not immediately seen to belong to $B$.

Theorem 26. The polynomials

$$
\begin{aligned}
& b_{e}=\sum_{i=2,4, \cdots, 2 n} \sum_{\sigma \in \boldsymbol{\Sigma}_{2 n}}(-1)^{\sigma} x_{\sigma(1)} \cdots x_{\sigma(i-1)^{x} 2 n+1} x_{\sigma(i)} \cdots x_{\sigma(2 n)}, \\
& b_{0}=\sum_{i=1,3, \cdots, 2 n+1} \sum_{\sigma \in \Sigma_{2 n}}(-1)^{\sigma} x_{\sigma(1)} \cdots x_{\sigma(i-1)^{x} 2 n+1} x_{\sigma(i)} \cdots x_{\sigma(2 n)}
\end{aligned}
$$

are identities of $F_{n}$.

Lemma 27. Let $f\left(x_{1}, \cdots, x_{k}\right)$ be a multilinear polynomial which vanis bes under every simple substitution $u=\left(a_{1}, \cdots, a_{k}\right)$ from $F_{n}$ such that $a_{k}=e_{r r}$ is idempotent. Then $f$ is an identity of $F_{n}$.

Proof. Since $f$ is multilinear it suffices to show that it vanishes under every simple substitution from $F_{n}$. Let a simple substitution $u=\left(a_{1}, \cdots, a_{k}\right)$ be given, for which $a_{k}=e_{r s}, r \neq s$. Then, using the assumption of the lemma, we may write $f\left(a_{1}, \cdots, a_{k-1}, e_{r s}\right)=f\left(a_{1}, \cdots, a_{k-1}, e_{r s}\right)+f\left(a_{1}, \cdots, a_{k-1}, e_{s s}\right)=$ $f\left(a_{1}, \cdots, a_{k-1}, e_{r s}+e_{s s}\right)$, and prove that the right member of the equation vanishes. Let $\tau$ be the similarity transformation of $F_{n}$ determined by $1+e_{r s}$ (this is a regular matrix whose inverse is $\left.1-e_{r s}\right)$. Then $r: e_{r s}+e_{s s} \mapsto e_{s s}$ and we have $f\left(a_{1}, \cdots, a_{k-1}, e_{\text {ts }}+e_{s s}\right)^{\tau}=f\left(a_{1}^{\tau}, \cdots, a_{k-1}^{\tau}, e_{s s}\right)=0$. (Remark: $\left(a_{1}^{\tau}, \cdots\right.$, $\left.a_{k-1}^{\tau}, e_{s s}\right)$ is not, in general, a simple substitution. However, it is a sum ot simple substitutions, all of which have $e_{s s}$ for the last term and thus annihilating f). But $\tau$ is monomorphism so we may conclude that $f\left(a_{1}, \cdots, a_{k-1}, e_{r s}+e_{s s}\right)$ $=0$. 
Notation. $M_{\sigma, i}=x_{\sigma(1)} \cdots x_{\sigma(i-1)} x_{2 n+1} x_{\sigma(i)} \cdots x_{\sigma(2 n)}$, for $\sigma \in \Sigma_{2 n}$ and $1 \leq i \leq 2 n+1$.

Proof of Theorem 26. We shall prove that $F_{n}$ satisfies the identities $b_{0}+$ $b_{e}$ and $b_{0}-b_{e}$, from which the conclusion obviously follows. The case of $b_{0}-$ $b_{e}$ is easy, for $b_{o}-b_{e}=s_{2 n+1}$ and this is an identity of $F_{n}$. We proceed to show that $F_{n}$ satisfies the identity $b_{o}+b_{e}$, which may be written in the form $b_{o}+b_{e}=\Sigma_{\sigma \in \Sigma_{2 n}}(-1)^{\sigma} \sum_{i=1}^{2 n+1} M_{\sigma, i}$. By Lemma 27 it is enough to show that this polynomial vanishes under every simple substitution $u=\left(a_{1}, \cdots, a_{2 n+1}\right)$, for which $a_{2 n+1}=e_{r r}$. To this end, we apply the Amitsur-Levitzki theorem and obtain $P \equiv s_{2 n}\left(a_{1}, \cdots, a_{2 n}\right)=\Sigma_{\sigma \in \Sigma_{2 n}}(-1)^{\sigma} a_{\sigma(1)} \cdots a_{\sigma(2 n)}=0$. Define $A_{k l}=$ $\left\{\sigma \in \Sigma_{2 n} \mid a_{\sigma(1)} \cdots a_{\sigma(2 n)}=e_{k l}\right\}$, and note that $\Sigma_{\sigma \in A}(-1)^{\sigma} a_{\sigma(1)} \cdots a_{\sigma(2 n)}=$ $t_{k l} e_{k l}$ for a suitable $t_{k l} \in F$. Since

$$
P=\sum_{k, l=1}^{n} \sum_{\sigma \in A_{k l}}(-1)^{\sigma} a_{\sigma(1)} \cdots a_{\sigma(2 n)}=\sum_{k, l=1}^{n} t_{k l} e_{k l}=0,
$$

we conclude that $t_{k l}=0$ for all $k, l$. Consider any $\sigma$ for which $a_{\sigma(1)} \cdots a_{\sigma(2 n)}$ $\neq 0$. The sequence $\left(a_{\sigma(1)}, \cdots, a_{\sigma(2 n)}\right)$ must then have the form $\left(e_{i_{1} i_{2}}, e_{i_{2} i_{3}}\right.$, $\left.e_{i_{3} i_{4}}, \cdots, e_{i_{2 n} i_{2 n+1}}\right)$, and we let $\nu_{\sigma}$ denote the number of occurrences of the number $r$ in the derived sequence $\left(i_{1}, i_{2}, \cdots, i_{2 n+1}\right)$. We claim that if two permutations $\sigma, \tau$ belong to the same $A_{k l}$ then $\nu_{\sigma}=\nu_{\tau}$. There are several cases to consider and we treat, for example, the case where $r \neq k, l$. In this case $\nu_{\sigma}$ is simply half the number of occurrences of $r$ in the indices of the unit matrices $a_{1}, \ldots$, $a_{2 n}$, and this number does not depend on the order of the matrices, hence must be the same for all permutations in $A_{k l}$. A similar argument can be given in the cases where $r$ is equal to one of $k, l$ or both. Denote the common value of $\nu_{\sigma}$ for all $\sigma \in A_{k l}$ by $\nu_{k l}$. Notice that $\nu_{\sigma}=\nu_{k l}$ is precisely the number of places in the product $a_{\sigma(1)} \cdots a_{\sigma(2 n)}$, where the factor $a_{2 n+1}=e_{r r}$ can be inserted without annihilating the product (and, of course, without altering its value). Therefore

$$
\begin{aligned}
Q_{k l} & \equiv \sum_{\sigma \in A_{k l}}(-1)^{\sigma} \sum_{i=1}^{2 n+1} a_{\sigma(1)} \cdots a_{\sigma(i-1)^{2 n+1}} a_{\sigma(i)} \cdots a_{\sigma(2 n)} \\
& =\sum_{\sigma \in A_{k l}}(-1)^{\sigma} \nu_{k l} a_{\sigma(1)} \cdots a_{\sigma(2 n)}=\nu_{k l} t_{k l} e_{k l}=0 .
\end{aligned}
$$

But $\left(b_{o}+b_{e}\right)\left(a_{1}, \cdots, a_{2 n+1}\right)$ is just the sum of all the $Q_{k l}$ 's and so must vanish, that is, $b_{o}+b_{e}$ is an identity of $F_{n}$.

Theorem 20 may now be used to obtain 
Corollary 28. The identities $b_{o}$ and $b_{e}$ belong to B, i.e. they follow from the standard identity $s_{2 n}$.

Remark. In the $i$-summation defining $b_{0}$, the first and last terms are clearly identities of $F_{n}$, obtained from $s_{2 n}$ by multiplying on the left and right (respectively) by $x_{2 n+1}$. Therefore the following polynomial is also an identity of $F_{n}$ :

$$
\sum_{i=3,5, \cdots, 2 n-1} \sum_{\sigma \in \boldsymbol{\Sigma}_{2 n}}(-1)^{\sigma} x_{\sigma(1)} \cdots x_{\sigma(i-1)} x_{2 n+1} x_{\sigma(i)} \cdots x_{\sigma(2 n)}
$$

Acknowledgement. This work forms part of my $\mathrm{Ph} . \mathrm{D}$. thesis written in the Hebrew University of Jerusalem. I wish to express my warm thanks to my thes is advisor Professor S. A. Amitsur.

Added in proof. A complete analys is of the multilinear identities of $F_{2}$ was carried out by A. A. Klein and S. Rosset, using a computer. It turns out that the dimension of these identities is 29 , and they all follow from the standard identity $s_{4}$ and the identity $\left[\left[x_{1} x_{2}\right]\left[x_{3} x_{4}\right]+\left[x_{3} x_{4}\right]\left[x_{1} x_{2}\right], x_{5}\right]$. Similiar results were brought to my attention by $\mathrm{R}$. Kruse.

\section{REFERENCES}

1. S. A. Amitsur and J. Levitzki, Minimal identities for algebras, Proc. Amer. Math. Soc. 1 (1950), 449-463. MR 12, 155.

2. I. N. Herstein, Noncommutative rings, Carus Math. Monographs, no. 15, Math. Assoc. Amer.; Wiley, New York, 1968. MR 37 \#2790.

3. N. Jacobson, Structure of rings, 2nd rev. ed., Amer. Math. Soc. Colloq. Publ., vol. 37, Amer. Math. Soc., Providence, R. I., 1964. MR 36 \#5158.

DEPARTMENT OF MATHEMATICS, HEBREW UNIVERSITY, JERUSALEM, ISRAEL

DEPARTMENT OF MATHEMATICS, UNIVERSITY OF OREGON, EUGENE, OREGON 97403

Current address: Department of Mathematics, University of California, Los Angeles, California 90024 